\title{
Dynamic brain imaging: Event-related optical signal (EROS) measures of the time course and localization of cognitive-related activity
}

\author{
GABRIELE GRATTON and MONICA FABIANI \\ University of Missouri, Columbia, Missouri
}

\begin{abstract}
This paper describes the concept of dynamic brain imaging and introduces a new methodology, the event-related optical signal, or EROS. Dynamic brain imaging allows one to study noninvasively the time course of activity in specific brain areas. Brain imaging data can contribute to the analysis of the subcomponents of the human information processing system and of their interactions. Several brain imaging methods provide data that possess spatial and temporal resolution at various degrees and can be used for this purpose. In this paper, we focus on the EROS method, which yields data with millisecond temporal resolution and subcentimeter spatial resolution. We describe the fundamentals of this method and report several examples of the types of data that can be obtained with it. Finally, we discuss the possibility of combining different imaging methods, as well as the advantages and limitations that can be expected in this process.
\end{abstract}

In the last decade, there has been a large growth of interest about the brain mechanisms that are associated with a number of psychological functions, including vision, hearing, movement preparation and execution, attention, memory, language, and emotions, among many others (Gazzaniga, 1995). The study of these mechanisms can be carried out at several different levels, from the molecular to the systemic (Marr, 1982). For each of these different levels, there are research instruments and approaches that are often specific to that level and vary along a number of dimensions, including invasiveness and spatial and temporal specificity (Churchland \& Sejnowski, 1988).

A major thrust of much of this research has been the development of noninvasive functional brain imaging methodologies with good spatial and temporal resolution, which allow investigators to study, more or less directly, the activity of the intact human brain (Posner \& Raichle, 1994; Toga \& Mazziotta, 1996). In this paper, we will review and discuss the role of imaging methods and their integration with psychological models of cognition. The focus will be on functional imaging techniques and, particularly, on their spatial and temporal resolution characteristics. Specifically, we will (1) consider the contexts in which functional imaging methods may be useful for the study of psychological functions, (2) briefly review and

Preparation of this paper was supported in part by NIMH Grant MH57125 to G.G. and by McDonnell-Pew Grant 97-32 to M.F. The authors wish to thank Nelson Cowan, Steve Hackley, Toshinori Kato, Kathleen McDermott, Mike Stadler, Endel Tulving, Arno Villringer, and one anonymous reviewer for comments and suggestions on an earlier version of this manuscript. Correspondence should be addressed to G. Gratton, Department of Psychology, University of Missouri, 210 McAlester Hall, Columbia, MO 65211-2500 (e-mail: grattong@ missouri.edu) discuss the properties of the most widely used imaging methods, (3) introduce a new technology (the eventrelated optical signal, or EROS) that provides data with a good combination of spatial and temporal resolution, (4) outline some examples of the data that have been obtained with EROS to date, and (5) discuss the utility of combining imaging methods.

\section{WHY IS FUNCTIONAL BRAIN IMAGING RELEVANT TO THE STUDY OF PSYCHOLOGICAL FUNCTIONS?}

It has been known for more than 100 years that lesions to particular areas of the brain bring forth specific types of neurological deficits (e.g., Broca, 1865). These data, as well as a number of other observations carried out with a variety of methodologies, clearly indicate that the brain can be viewed as a complex information processing machine comprising structures or circuits specialized for different types of psychological functions. For instance, areas of the occipital lobe appear to be specialized for vision, and areas of the temporal lobe appear to be specialized for hearing. It has also been clear for a long time that different areas work together to produce complex behavioral functions that may be disrupted if connections among areas are lesioned (e.g., disconnection syndromes, such as split brain; for a review, see Kolb \& Whishaw, 1996).

In parallel with (and, to a fair degree, independently from) the development of knowledge about the functional specialization of different brain areas, cognitive psychologists have developed complex models of the human information processing system, as well as experimental paradigms to test them. These models postulate the existence a number of different subprocesses-each specialized in some type of elementary transaction-interacting in a 
complex fashion to produce final overt behavior (e.g., Sanders, 1981; Sternberg, 1969; see also Gardner, 1985, and Posner, 1989). These cognitive models have been, by and large, developed without reference to the brain; however, in the last two decades, both cognitive psychologists and neuroscientists have increasingly begun to incorporate each others' data in the development of their theories of mind/brain function. This has brought forth the development of a new discipline, cognitive neuroscience (see Gazzaniga, 1995; Gazzaniga, Ivry, \& Mangun, 1998; Rugg, 1997).

Within the broad realm of cognitive neuroscience, noninvasive functional brain imaging methods have played a pivotal role, as they allow researchers to derive measures of physiological activity from the human brain in experimental conditions that closely parallel those that are used for behavioral studies of information processing. Two main research traditions have converged into the domain of functional neuroimaging. One is the mental chronometry approach widely used by cognitive psychologists, in which the speed of mental processes and their relative order of occurrence are the critical variables of interest. The other is the investigation of the function of specific cortical areas, which is typically the focal interest of researchers in neuropsychology. The first approach emphasizes temporal information, whereas the second emphasizes spatial information. An integration of the two approaches into the domain of cognitive neuroscience requires the development of brain imaging methods with both high spatial and temporal resolution or the combination of existing techniques that emphasize different types of information.

To a large extent, imaging methods are useful to psychologists because they allow them to break down observations obtained on the whole system (i.e., traditional behavioral measures, such as reaction time and accuracy) into measures of subcomponents of the system. In other words, imaging data can be used to monitor intermediate stages of the information processing system in order to determine the nature of its basic components (or elements) and the ways in which they interact (Meyer, Osman, Irwin, \& Yantis, 1988). For this to be possible, the physiological measures must possess some degree of spatial, temporal, and/or functional specificity-that is, they need to provide information about which neural structures are active during a particular task, when they are active, and under what experimentally specified conditions they are active.

Several recent publications exemplify the integration of behavioral and imaging data. For instance, G. Gratton, Coles, Sirevaag, Eriksen, and Donchin (1988) identified a measure of the event-related brain potential (ERP) associated with response preparation, the lateralized readiness potential (LRP). They used the LRP to monitor the time course of response activation in certain types of conflict paradigms (i.e., in cases in which two pieces of information, leading to different responses, are presented simultaneously). They found that there was a temporary activation of the incorrect response, followed by the activation of the correct response within the same trial. G. Gratton et al. (1988) used these data to argue in favor of a continuous flow model of information processing, in contrast with serial stage models.

Other imaging studies have investigated the involvement of the two hemispheres in encoding and retrieval processes. Kelley et al. (1998) used functional magnetic resonance imaging (fMRI) to show that symmetrical cortical areas in the two hemispheres were used during encoding tasks that differed in the type of information (words, pictures, or faces) to be memorized: Words activated an area in the left hemisphere, faces activated the same area in the right hemisphere, and pictures activated the area bilaterally. This type of information could be used to argue in favor of models emphasizing the separate contributions of the two hemispheres to the encoding of different types of stimuli. A different role of the two hemispheres in encoding and retrieval was proposed by Tulving, Kapur, Craik, Moscovitch, and Houle (1994; see also Buckner, 1996, and Nyberg, Cabeza, \& Tulving, 1996). Nyberg et al. used positron emission tomography (PET) data to argue that encoding and retrieval involve both general and specific processes. In another study, Fiez et al. (1996) used PET to determine the brain areas involved in the short-term maintenance of linguistic information. They found that prefrontal regions were active during periods requiring the maintenance of both verbal and nonverbal information, whereas left frontal opercular regions were involved specifically in the rehearsal of verbal material. These data may be used as evidence in favor of models in which separate mechanisms are postulated for the maintenance of verbal material.

In another set of studies, neuroimaging work was used to refine and discriminate among cognitive models of attention. Heinze et al. (1994; see also G. Gratton, 1997) found that early selective attention for visual stimuli can be demonstrated in extrastriate cortex in humans ( 80 $130 \mathrm{msec}$ after stimulus onset), but not in primary visual cortex. These findings support early selection models of attention. Petersen, Corbetta, Miezin, and Shulman (1994; see also Corbetta, Shulman, Miezin, \& Petersen, 1995) found differences in the areas that are activated in tasks requiring tonic attention to various features, the conjunction of features, and attentional shifts, suggesting that different types of attention may tap into different brain systems. In addition, Rees, Frith, and Lavie (1997) found that motion processing of irrelevant stimuli was reduced (as indicated by a reduction of activity in area $V 5$ ) during a high-load condition of the relevant (linguistic) task.

Similarly, much research has recently focused on false memory, which occurs when a person recalls or recognizes an event that actually never happened (Loftus \& Pickrell, 1995; Roediger, 1996; Roediger \& McDermott, 1995). False and true memories appear fairly indistinguishable behaviorally. Therefore, researchers have begun investigating the existence of brain indices that might enable one to distinguish between them (Johnson et al., 


\section{Behavioral Output, ANS Measures} ERPs
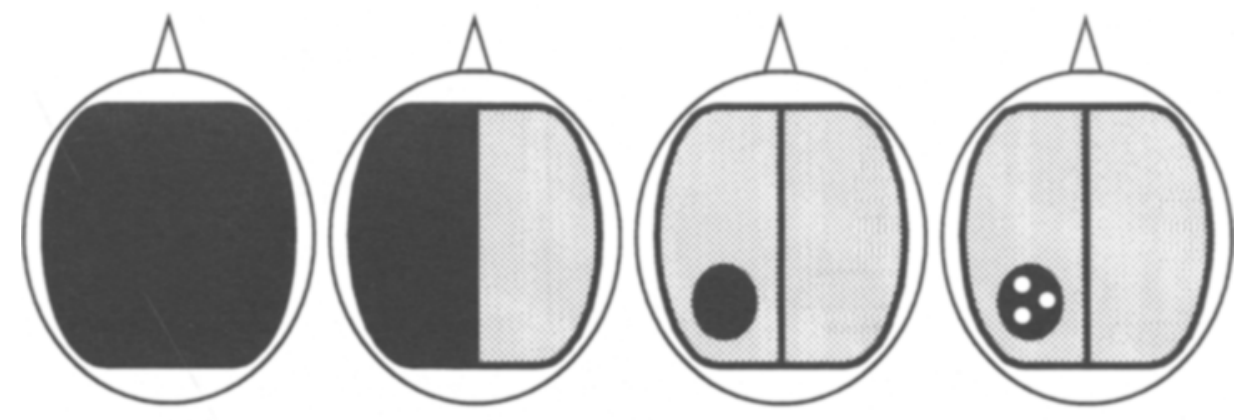

Low

\section{Spatial specificity}

Figure 1. Schematic representation of the spatial specificity of several noninvasive techniques for studying the activity of the human brain. The techniques are classified on the basis of the level of specificity of the brain area that could be considered responsible for the observed variance. This varies from measurements that can be influenced, at least in principle, by activity of the whole brain up to measures that can be considered specific to particular locations within a functional area. For several techniques, the level of spatial specificity is not sharply defined. An example is given by ERPs and MEG, for which the spatial specificity varies depending on the type of measurement procedure adopted and the particular activity that is investigated.

1997; Schacter et al., 1996). Although this research is not yet conclusive, it is an instance in which dissociations between brain activity and overt behavior may help constrain and refine theoretical models of memory.

\section{SPATIAL AND TEMPORAL RESOLUTION}

There are several brain imaging measures with limited or no invasiveness that satisfy at least some of the criteria of spatial and temporal specificity outlined above and that can therefore be used to determine which areas of the brain are active during particular psychological tasks. These techniques are collectively labeled functional brain imaging methods, and they have recently received a large amount of attention in specialized journals and in the media (e.g., Barinaga, 1997). The two best known and most widely used methods are PET (Raichle, 1986, 1994) and fMRI (Bandettini, Wong, Hinks, Tikofsky, \& Hyde, 1992; Belliveau et al., 1991; Frahm, Bruhn, Merboldt, \& Hänicke, 1992; Kwong et al., 1992; Turner, 1995). In addition, other physiological techniques are also available that, while limited in spatial specificity, provide very detailed temporal information about brain activity. These include ERPs (for reviews, see Fabiani, G. Gratton, \& Coles, in press, and Rugg \& Coles, 1995) and magnetoencephalography (MEG; Hari \& Lounasmaa, 1989).

For both spatial and temporal dimensions, it is possible to identify different types of information that can be obtained with each methodology. For instance, within the spatial dimension, different techniques can provide information about activity in the brain as a whole (minimum level of spatial specificity), the relative activity in the two hemispheres, activity in specific areas in each hemisphere, or the general principles of functional organization within an area (which, at present, is the maximum level of spatial specificity that can be obtained with noninvasive methodologies; see Figure 1). A similar logic can be applied to classify techniques in the temporal domain: Techniques may provide information about the general level of activity during an experimental session (minimum level of temporal specificity), during a block of trials, during a particular trial, or about the time course of activation within a particular trial (maximum level of temporal specificity; see Figure 2).

The most common noninvasive imaging techniques can be classified into two broad groups. The first group comprises measures that rely on hemodynamic changes (e.g., increased blood flow) that occur in areas of the brain that are active. These include PET (in particular, $\mathrm{O}^{15}$-PET) and fMRI (in particular, blood-oxygenation-leveldependent, or BOLD, fMRI). 2 The basis for these signals is the observation that hemodynamic changes in response to neuronal activity are quite localized and can therefore yield very specific spatial information (Engel et al., 1994: Frostig, Lieke, Ts'o, \& Grinvald, 1990; Malonek \& Grinvald, 1996; Raichle, 1986; Roy \& Sherrington, 1890; 


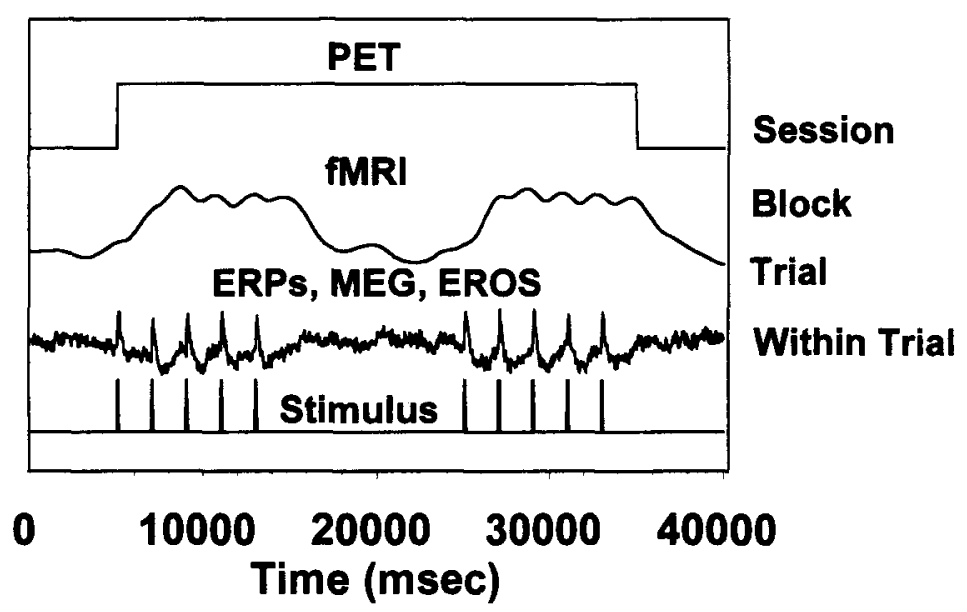

Figure 2. Schematic representation of the time course and temporal specificity of several noninvasive techniques for studying the activity of the human brain. The example is based on a situation in which two series (blocks) of five stimuli (trials) are presented in succession every 2 sec. The stimuli represent individual trials in a cognitive experiment in which subjects may be required to perform a choice reaction-time task. The total period investigated may last $40 \mathrm{sec}$ (session). Procedures vary in their degree of temporal specificity, with some providing only one piece of information across the whole session, whereas others may provide information about the time course of the response within each particular trial.

Villringer, 1997; Villringer \& Dirnagl, 1995). With these measures, it is possible to obtain signals that are specific to particular areas (i.e., signals are localized within a few millimeters of tissue), and, in some cases, it is also possible to obtain maps of the functional organization within areas (e.g., DeYoe et al., 1996; Reppas, Niyogi, Dale, Sereno, \& Tootell, 1997; Sereno et al., 1995; Tootell, Dale, Sereno, \& Malach, 1996). This spatial information can be used to decompose different aspects of information processing, as indicated by the imaging research on motor preparation, working memory, attention, and false memory outlined above. Hemodynamic techniques, however, are limited in terms of their temporal specificity, mainly because the hemodynamic effect (i.e., increase in blood flow) develops slowly, with its onset occurring about $2 \mathrm{sec}$ after a brain area becomes active and its peak occurring 5-10 sec thereafter (Malonek \& Grinvald, 1996). In addition, some of these measures require an extended period to be obtained (e.g., PET measures). For these reasons, hemodynamic methods can provide information about the activity during a session, during a block of trials, or, at best, during individual trials (see Figure 2). In the latter case, a procedure for deconvolving the overlapping effects of consecutive stimulations is required (e.g., Buckner et al., 1996; for attempts at within-trial discrimination, see also J. D. Cohen et al., 1997, Courtney, Ungerleider, Keil, \& Haxby, 1997, and Dale \& Buckner, 1997).

The second group of techniques includes measures based on electrophysiological phenomena, thought to be associated with neuronal activity. The bases of these mea- sures are the electrical currents associated with the movement of ions that characterize depolarization and hyperpolarization of neurons. When a large number of neurons act synchronously and are spatially aligned ("open-field" configuration), the electrical and magnetic fields generated by individual neurons summate and can therefore be measured at the surface of the head (Allison, Wood, \& McCarthy, 1986). Techniques based on these phenomena include ERPs and MEG.

Because the propagation of the electric and magnetic fields to the surface is practically instantaneous, these methods can directly monitor the occurrence of electrophysiological events in the brain, and, therefore, they have excellent temporal resolution (i.e., they have the capability of determining the relative order of occurrence of electrical events in the brain). Data with high levels of temporal resolution can be particularly useful to discriminate among cognitive theories. For example, Hackley and Valle-Inclán (1998) used the LRP to discriminate between two models of the effects of an accessory stimulus (an irrelevant stimulus presented at the same time as the imperative signal) in a choice reaction time (RT) paradigm. They found that the reduced RT in the presence of the accessory stimulus was due to a speeding of perceptual and decision processes rather than to motor processes per se, as had been assumed on the basis of behavioral evidence (Sanders, 1981).

The major limitation of noninvasive electrophysiological techniques is that, because measures are taken at some distance from their origin, the effects of the electro- 
physiological activity generated in different brain areas are very difficult to distinguish from one another. Besides making it difficult to pinpoint the brain structures. that are responsible for the observed surface activity, this limitation has other undesirable consequences. One of them is that, because activities that originate in different areas often overlap in time, it is difficult to determine whether any observed ERP effect is to be attributed to one or more underlying structures (or ERP components). ${ }^{3}$ This limits the effective spatial specificity of electrophysiological measures and, in some cases, makes it difficult to draw conclusions that go beyond that of a differential involvement of the two hemispheres at a given point in time (see Figure 1). This problem is more pronounced with ERPs than it is with MEG, because magnetic fields propagate through the head in a more orderly fashion and tend to attenuate more with distance from the source. ${ }^{4}$

Until a few years ago, these problems were considered an insurmountable obstacle. Recently, several procedures (collectively labeled source-analysis algorithms) have been devised to increase the spatial specificity of electrophysiological measures (e.g., brain electrical source analysis, or BESA; Scherg \& Von Cramon, 1986). In some cases, this has allowed investigators to pinpoint the specific cortical area involved in the generation of a particular scalp-recorded activity (Clark \& Hillyard, 1996; Scherg, Vajsar, \& Picton, 1989; Winkler et al.,'1995). However, it has been difficult to generalize these procedures to all cases. In fact, the validity of the results depends on the validity of assumptions about the nature and configuration of the underlying sources (i.e., the brain structures responsible for the surface-recorded activity) used in the source analysis process. Basic knowledge about source configuration is more refined for early effects (such as the brain-stem auditory potentials) than for longer latency "cognitive" responses (see Miltner, Johnson, Simpson, \& Ruchkin, 1994). The latter case can sometimes be addressed by a combination of hemodynamic and electrophysiological methods (Heinze et al., 1994).

\section{THE ADVANTAGES OF COMBINING SPATIAL AND TEMPORAL RESOLUTION}

So far, we have focused on the use of spatial and temporal information about brain activity to break down the response of the whole system into its subcomponents. We have emphasized that this decomposition can be based on (and take advantage of) either spatial or temporal information. Existing brain imaging methods usually trade off between these two types of information. However, there are several questions about the information processing system (and its brain implementation) that can be better addressed by methodologies combining both spatial and temporal specificity.

One of these questions, often central to understanding the dynamics of information processing, concerns the relative order in which different processes occur. An imag- ing technique combining spatial and temporal specificity could be used to determine whether a particular brain area is activated (or inhibited) before another. To the extent that there is an association between activity in a particular brain area and the occurrence of a particular psychological operation (or event), it is possible to use this information to draw conclusions about the ordering of different psychological processes. Within limits, information of this type can be obtained from data with little spatial specificity but high temporal resolution (such as ERPs); provided that separate measures can be obtained for different functional events. However, the addition of spatial resolution may greatly enhance the ability to separate the individual functional events and therefore considerably increase the analytical power. In contrast, measures with very limited temporal resolution provide little direct information about the order of processing, because activation of a particular area may occur at any time during processing. The studies of Heinze et al. (1994) and G. Gratton (1997) are examples of the advantages of combining spatial and temporal resolution to determine the level at which selective attention begins to operate within the visual system.

A combined spatiotemporal approach may be particularly useful in the study of the interactions and correlations among the activities of different brain areas. Because most cognitive operations are likely to result from interactions among different areas (see Mesulam, 1990; Squire, 1989), this information may be very important for obtaining a detailed description of the brain mechanisms responsible for cognitive operations. An example is given by the recent interest in the role of high-frequency oscillations of neuronal activity during information processing. Some recent theories (Gray, Konig, Engel, \& Singer, 1989; Roelfsema, Engel, Konig, \& Singer, 1996; Singer, 1994; Skarda \& Freeman, 1987) propose that information processing in several brain areas may be carried out through the modulation of some basic high-frequency activity (e.g., $40 \mathrm{~Hz}$ or gamma band) and that areas of the brain that are involved in processing the same stimuli are all activated at the same basic frequency. In this case, the network of areas involved in stimulus processing could be revealed by the coherence of the activity observed in different brain structures. However, this hypothesis is very difficult to test using measures that provide only spatial or temporal information. In the first case, the data may not provide enough temporal information for studying the dynamic interrelationships among areas. In the absence of spatial information, it is difficult to determine whether correlated (or coherent) activities observed at different locations reflect measures of correlated (but different) phenomena or reflect the same phenomenon volume-conducted at different locations (although, recently, there have been some attempts to address this issue; see Gevins, 1996; Menon et al., 1996; Thatcher, Toro, Pflieger, \& Hallett, 1994).

Another approach to obtaining optimal levels of spatial and temporal resolution involves the combination of more 


\section{Optical Methods}

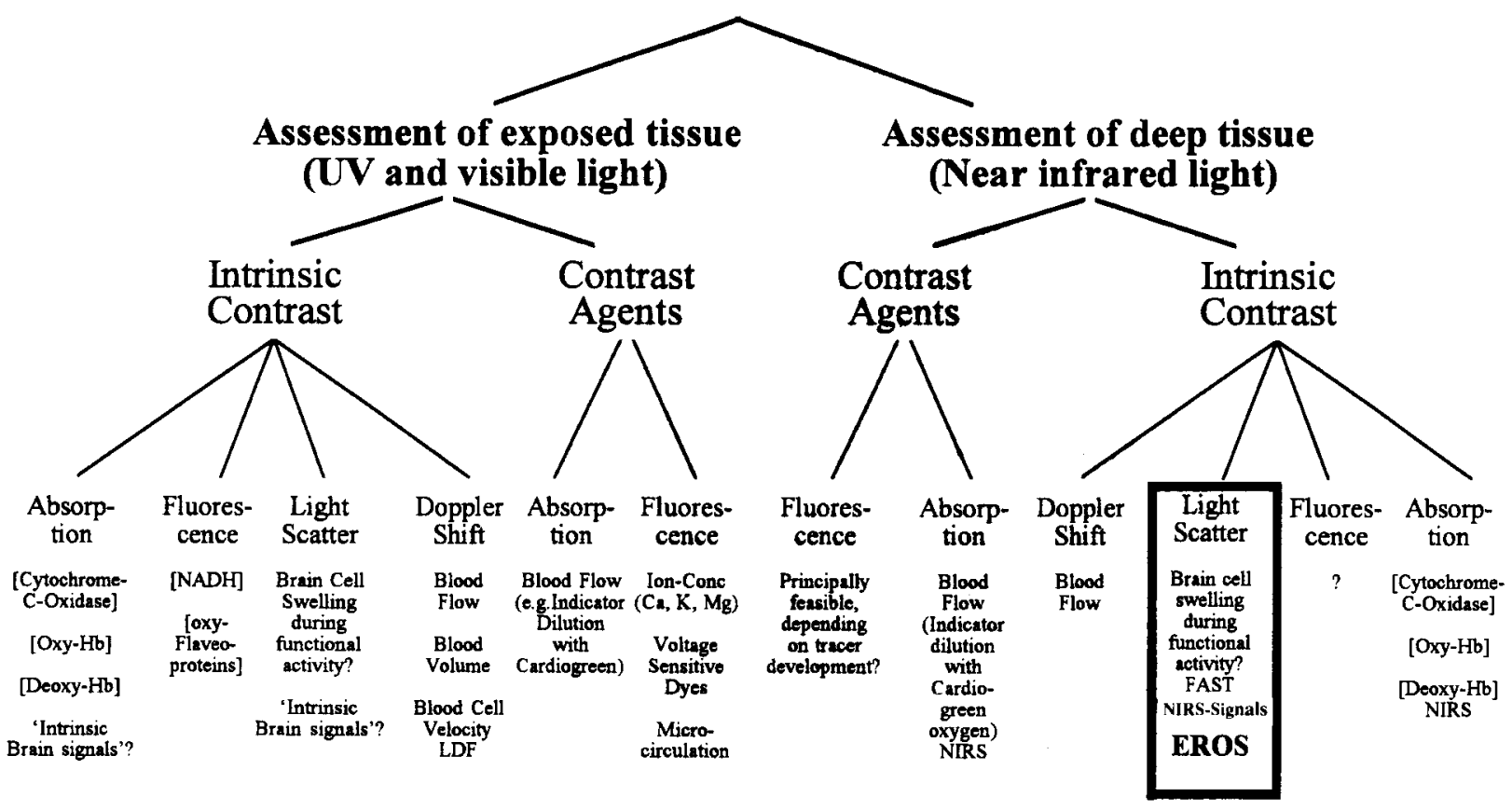

Figure 3. Classification of optical methods for studying brain function. Optical methods vary along a number of dimensions, including invasiveness, type of contrast agent used, and type of phenomenon investigated. In this paper, we focus on noninvasive measures based on intrinsic signals associated with changes in absorption (e.g., NIRS) or scattering (e.g., EROS). (This illustration is courtesy of Arno Villringer.)

than one imaging method. This strategy is reviewed later. In the following sections, we will describe a new imaging approach based on optical methods (EROS) that possesses a good combination of spatiotemporal specificity.

\section{OPTICAL METHODS}

EROS is one of many methodologies that are based on the measurement of changes in optical parameters of brain tissue (i.e., light absorption and scattering) when the tissue is active (see Figure 3). We will focus here on measures that are obtained on the intact brain (and therefore will not discuss studies based on microscopic measures taken on lab preparations).

Although relatively new, optical measures of the intact brain are becoming a widely used tool in neuroscience (Bonhoeffer \& Grinvald, 1996; Villringer \& Chance, 1997). The common denominator of all these measures is the use of light with a wavelength between 200 and $1,000 \mathrm{~nm}$ (i.e., ranging from the ultraviolet to the nearinfrared) to study changes in the scattering and absorption properties of tissue due to neuronal activity. By and large, absorption changes are related to variations in the concentration of particular chemicals in the brain. Scattering changes are often related to variations in the physical characteristics of the brain (e.g., swelling of cells or organelles. changes of reflectivity and/or coloration with temperature, etc.). Optical measures vary widely, along several dimensions (see Figure 3). We will discuss two of these dimensions in the next section of this paper. The first is the degree to which the measures can be taken on intact (unlesioned) subjects (i.e., the invasiveness of a measure). The second is the type of signal studied (e.g., intrinsic vs. extrinsic), and its relationship to neuronal activation.

\section{Types of Optical Measures}

Invasive optical imaging has become a standard tool for investigating the functional micro-architecture of the cortex, including its columnar organization (Bonhoeffer \& Grinvald, 1996; Grinvald, Lieke, Frostig, Gilbert, \& Wiesel, 1986; Malonek, Tootell, \& Grinvald, 1994). These measures are usually taken by exposing the cortex of anesthetized animals or by implanting optic fibers into various brain structures. They involve either (1) the observation of intrinsic signals (i.e., of the natural changes in brain tissue coloration and/or reflectivity in active areas of the brain) or (2) the observation of extrinsic signals (i.e., of changes of the optical properties of contrast agents - e.g., voltage-sensitive dyes-injected into brain tissue in response to stimulation).

The greatest advantage of these measures is the possibility of studying simultaneously extended sections of the cortex while maintaining a spatial resolution of 50 microns or better. This allows investigators to determine the way in which areas of the cortex are organized (e.g. 
the "pinwheel" organization of visual cortex; Bonhoeffer \& Grinvald, 1993). Recently, this work has been extended to other areas, such as the somatosensory cortex (Cannestra, Blood, Black, \& Toga, 1996), the inferotemporal cortex (Wang, Tanaka, \& Tanifuji, 1996), and the hippocampus (Rector, Poe, Kristensen, \& Harper, 1995, 1997). In humans, this approach is limited to studies conducted during surgical operations. For example, Haglund (1997; see also Haglund, Ojemann, \& Hochman, 1992) has reported optical studies of various cortical areas, including the motor cortex and language areas, conducted using a charge-coupled device camera in the operating room. These studies show that it is possible to characterize these areas during brain surgery, which may help surgeons guide their work without recourse to more invasive exploratory procedures (such as the use of implanted electrodes or electrical stimulation). However, to be of practical use, this application of optical imaging requires further technical advances and standardization (both in the recording methodology and in the brain activation protocols).

The noninvasive measurement of brain optical signals requires the use of near-infrared (NIR) light (see Appendix, section 1). The main obstacle to the passage of NIR light through the head is that most head tissues are scattering - that is, they diffuse light, sometimes to a very high degree (e.g., white matter). For this reason, the movement of NIR photons through the head is best described as random, similar to the diffusion of gas (Ishimaru, 1978). Some of the consequences of this phenomenon on the measurements of optical parameters will be described in the following sections.

\section{Physiological Bases of the Optical Signals}

The optical measures described in the remainder of this paper capitalize on the fact that the optical properties of the tissue itself may change with neuronal activity (intrinsic signal; Grinvald et al., 1986). Malonek and Grinvald (1996; see also Frostig, 1994; Frostig et al., 1990) identified several types of intrinsic signals in the exposed cortex of monkeys. First, there are scattering signals that may occur very rapidly when a brain area becomes active and subside very rapidly at the end of the activity. These signals are quite localized to the active region. The biophysics underlying this phenomenon is not entirely clear, but among possible causes are the movement of ions across the neuronal membrane (L. B. Cohen, 1972; Hill \& Keynes, 1949) and/or the reorientation of membrane proteins during the action potential (Stepnoski et al., 1991). In principle, these scattering changes could be used to monitor the activity of neurons in a noninvasive manner (i.e., as an alternative to microelectrode recording). Frostig et al. (1990; see also Federico, Borg, Salkauskus, \& MacVicar, 1994) demonstrated that scattering changes can also be observed at a more macroscopic level when bloodless hippocampal slices are subjected to tetanic activation. Recently, Rector et al. (1995) implanted fiber optics in the dorsal hippocampus of freely moving cats.
They observed reflectance changes during the cats' sleep, probably due to scattering phenomena, with a frequency similar to that of the electrical theta rhythm $(4-6 \mathrm{~Hz})$, which was recorded simultaneously with microelectrodes.

Andrew and MacVicar (1994) reported the effects of a number of osmolar, pharmacological, and electrophysiological manipulations on intrinsic optical signal changes in hippocampal slices that were also presumably due to scattering. Manipulations influencing the osmolar properties of the medium resulted in large changes in the intrinsic signal. Similar but smaller amplitude changes were observed when the hippocampal slices were electrically stimulated. The pattern of electrophysiological and pharmacological effects led Andrew and MacVicar to conclude that the intrinsic optical signal observed in hippocampal slices "primarily reveal glial and neuronal swelling associated with excitatory synaptic input and potential discharge. The signal can be imaged in real time to reveal neuronal activation, not only among hippocampal areas, but among neuronal regions" (p. 371).

In summary, there is evidence of changes in scattering in the hippocampus with temporal parameters similar to those of electrical activity. However, more research is needed to extend these conclusions to other cortical areas and to noninvasive measures in humans. In the remainder of this paper, we will review a series of noninvasive studies of human brain activity in which the recorded signal shares a number of properties with the intrinsic signal reported in these animal studies (both in terms of the optical changes observed and in terms of their relationship with electrophysiological measures). We label these intrinsic scattering effects fast optical effects, because they occur rapidly (i.e., within milliseconds after stimulation) and show a good temporal correspondence with electrical activity.

In addition to the scattering effects discussed so far, Malonek and Grinvald (1996; see also Frostig et al., 1990) also described absorption changes associated with variations in the concentration of oxyhemoglobin and deoxyhemoglobin in active brain areas. We label these phenomena slow optical effects, because their time course is on the order of seconds. The earliest of these phenomena is a small deoxygenation effect that begins approximately $500 \mathrm{msec}$ after neuronal activation, is very localized (within a 1-2-mm radius around the presumed active cortical area), and subsides within a few seconds from the cessation of activity. They propose that this signal is related to the passage of oxygen from the red cells to the tissue. However, this signal is very small and probably difficult to observe with noninvasive methods (but see Ernst \& Hennig, 1994).

The slowest type of intrinsic signal reported by Malonek and Grinvald (1996) is a large increase in oxygenation that begins about $1.5 \mathrm{sec}$ after neuronal activity occurs in a cortical area and subsides several seconds after the termination of the activity. This signal is related to vasodilation and is consistent with the widely reported increase in blood flow in active cortical areas (see Bel- 
liveau et al., 1991; Fox \& Raichle, 1986; Kwong et al., 1992; Roy \& Sherrington, 1890; Turner, 1995). Malonek and Grinvald (1996) described this signal as the least localized of the various intrinsic signals - -the affected area appears to extend $3-5 \mathrm{~mm}$ around the activated area. Still, this signal is sufficiently localized for most brain imaging applications (in which functional areas, or major subdivisions of these areas, are considered). ${ }^{5}$

In summary, both scattering and absorption changes are typically observed during neuronal activity. Scattering changes (fast optical effects) may be directly related to neuronal activity and are likely to be due to changes in the physical properties of neurons and other cells (e.g., swelling). Absorption changes (slow optical effects) are correlated with the hemodynamic effects observed with other imaging methods, such as PET and fMRI.

\section{Near-Infrared Spectroscopy}

As outlined in the previous section, optical imaging allows investigators to collect both neuronal and hemodynamic data, often in parallel and within the same experimental session. In this section, we briefly review one of the most widely used noninvasive applications of optical imaging, near-infrared spectroscopy (NIRS). The purpose of NIRS is the in vivo quantification of the concentration of significant substances through the use of NIR light of different wavelengths (see Villringer \& Chance, 1997; see also Jobsis, 1977). This technique is made possible by the observation that several substances of metabolic importance, including oxyhemoglobin, deoxyhemoglobin, and cytochrome-C-oxidasis, have characteristic and distinctive absorption spectra in the NIR range (see Appendix, sections 1 and 2).

NIRS is the only available method for measuring the absolute concentration of oxyhemoglobin and deoxyhemoglobin in a selected area of the body in a noninvasive fashion. In fact, $\mathrm{O}^{15}$-PET is a measure of blood flow, and the BOLD-fMRI signal is a function of the local deoxyhemoglobin concentration. Other advantages of NIRS are its low cost (relative to other regional measures of oxygenation and blood flow), its noninvasiveness, the possibility of recording data practically continuously, and the portability of the instrumentation that allows for bedside (and within-lab) recording. A further practical advantage of NIRS is its great simplicity of recording (at least with respect to fMRI and PET), which can make it very appealing for small research laboratories. A limitation may be that this technique is best suited to study signals from cortical areas, whereas subcortical areas may be difficult to study (the problems of penetration with a related measure, EROS, are discussed later).

Several investigators have used NIRS to measure noninvasively regional changes in the concentration of oxyhemoglobin and deoxyhemoglobin in the brain during visual stimulation (Kato, Kamei, Takashima, \& Ozaki, 1993; Meek et al., 1995; Wenzel et al., 1996), somatosensory stimulation (Obrig et al., 1996), motor tasks (Hirth. Obrig, Valdueza, Dirnagl, \& Villringer, 1997), and cog- nitive tasks (Chance, Luo, Nioka, Alsop, \& Detre, 1997; Hock et al., 1997; Hoshi \& Tamura, 1993). A typical finding is reported in Figure 4 (from Villringer \& Chance, 1997). This figure shows that a period of visual stimulation causes an increase in the concentration of oxyhemoglobin and a parallel decrease in the concentration of deoxyhemoglobin in occipital brain areas. These phenomena begin a few seconds after the onset of stimulation and last until a few seconds after the termination of stimulation. These data are consistent with the time course of the BOLD-fMRI signal under similar stimulation conditions. This is to be expected, because the BOLD-fMRI signal is likely to be due to the decrease in the concentration of deoxyhemoglobin in active brain areas. Hoshi and Tamura (1993) recorded NIRS simultaneously from different areas of the head and showed that the location at which the signal was the largest changed systematically in a fashion predictable on the basis of known principles of brain localization. Benaron and Villringer (1998) used recording and analysis procedures designed to allow the estimation of the location of the NIRS signal inside the head during a motor task (finger tapping) and found a good degree of correspondence between the localization of the NIRS signal and that of the BOLD-fMRI response for a similar condition.

These data suggest that NIRS can be used to study hemodynamic phenomena associated with brain activity. This technique possesses some degree of localization, although not nearly as good as that obtained with $\mathrm{fMRI}$. Procedures for improving the spatial specificity of the signal are presently under development. The temporal information that can be derived with NIRS is limited by the speed of the hemodynamic events under study: At best, they can afford the separation of the activity associated with each individual trial. Whether or not this procedure will become widely used by psychologists remains to be seen and will in part depend on the availability of threedimensional (3-D) reconstruction algorithms. A practical application for which NIRS has already provided useful results is an improvement in our understanding of the BOLD-fMRI signal (Villringer \& Dirnagl, 1995). Another possible niche for this methodology is the study of hemodynamic brain signals in children or other special populations for which use of other techniques, such as fMRI or PET, may not be practical and/or advisable (e.g., Benaron et al., 1990; Cooper et al., 1996; Stevenson, Vreman, \& Benaron, 1996).

\section{The Event-Related Optical Signal (EROS)}

Another noninvasive method for studying functional brain activity is EROS. Its major advantages are a combination of spatial and temporal specificity that permits the derivation of measures of the time course of activity in localized cortical areas, as well as low cost, portability, and compatibility with other techniques. The spatial specificity of EROS is on the order of a few millimeters (i.e., at the level of functional cortical areas or of their major subdivisions). Its temporal specificity is a few mil- 


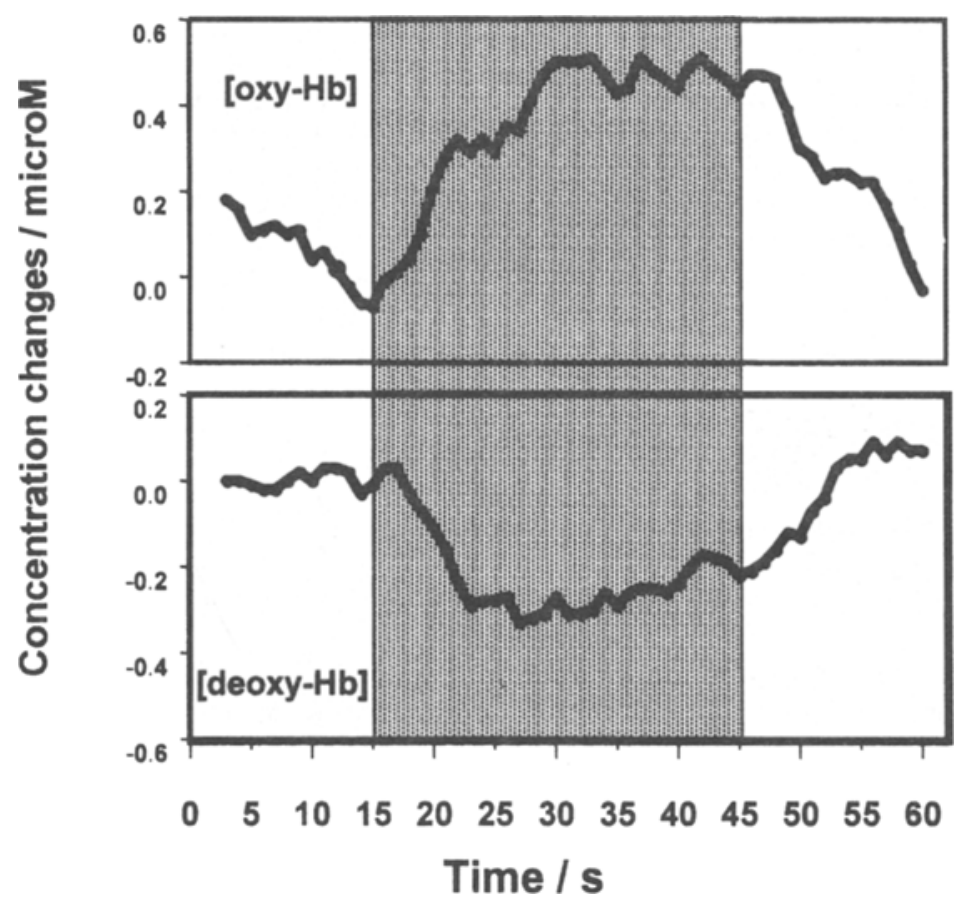

Figure 4. Example of the time course of changes in the concentration of oxyhemoglobin (top panel) and deoxyhemoglobin (bottom panel) during a photic stimulation interval (Second 15 to Second 45). Note the parallel increase in the concentration of oxyhemoglobin and decrease in the concentration of deoxyhemoglobin. This may be accounted for by an increase in blood flow, which introduces new oxygenated blood and flushes out deoxygenated blood from active brain areas. Note-From "Non-Invasive Optical Spectroscopy and Imaging of Human Brain Function," by A. Villringer and B. Chance, 1997, Trends in Neurosciences, 20, p. 439. Copyright 1997 by Elsevier Science. Reprinted with permission.

liseconds, providing a good picture of the time course of activity in specific cortical areas and potentially enabling investigators to study issues, such as the order of recruitment of different brain areas and their interrelations.

EROS measurements are conducted by using a light source that illuminates a small area on the surface of the head. The light source is usually a light-emitting diode (LED) or a laser diode that emits light in the NIR range. ${ }^{6}$ The light diffuses through the tissue, and some of it eventually exits the head at the location at which a detector (an optic fiber bundle) is located (usually at a few centimeters distance from the source; see Figure 5). This arrangement is similar to that used for NIRS measures and can be repeated for a number of source-detector pairs.

EROS measures differ in several respects from NIRS measures. The main difference is that with EROS the emphasis is on "fast" effects, with a time course of milliseconds. These effects are not likely to be due to changes in the concentration of major absorbers in the brain (e.g., oxyhemoglobin, deoxyhemoglobin, and water). Rather, probable targets for the EROS effects are changes in scattering properties, which, as we have seen earlier, can occur very rapidly in active cortical'areas.
To observe changes that occur very rapidly, a very fast data acquisition rate is used, with data recorded every few milliseconds. In some of our most recent studies, data are obtained every $20 \mathrm{msec}$, but the theoretical limit is much shorter (less than $1 \mathrm{msec}$ ). This allows one to obtain measures that can follow quite closely changes in the optical properties of the brain.

Because, unlike with NIRS, the emphasis is on scattering rather than absorption effects, EROS measures are usually not expressed in terms of changes in the concentration of target substances, and a spectroscopic approach is not required. In the approach used in our laboratory, light of a single NIR wavelength is used (in our earlier studies, $715 \mathrm{~nm}$; most recently, $750 \mathrm{~nm}$ ). ${ }^{7}$

Another major difference between the apparatus used in most NIRS studies and that employed in EROS studies is the use of time-resolved measures, rather than continuous measures (Cope \& Delpy, 1988; E. Gratton, G. Gratton, Fabiani, \& Corballis, 1996). These measures allow investigators to determine the time taken by photons to migrate between the source and the detector. For this reason, these measures are also called photonmigration measures. The main characteristics and ad- 

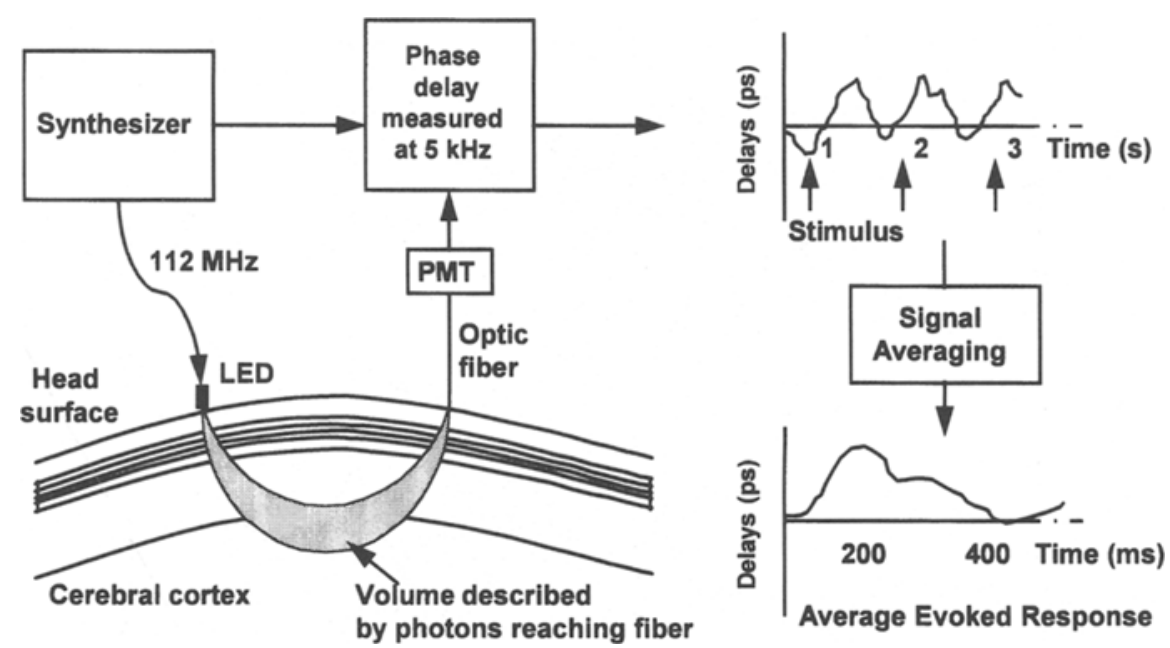

\begin{abstract}
Figure 5. Methods used for recording the event-related optical signal (EROS). The left panel shows a diagram of the recording apparatus, consisting of a radio-frequency synthesizer driving a light source (LED or laser diode), whose modulated light is injected into the head. Some of the light is picked up from a detector (an optic fiber connected to a photomultiplier tube, or PMT). The output of the PMT is used to compute quasi-continuously the parameters of migration of photons through the head (light attenuation, or intensity, and phase delay, or time of flight). The right panel shows how these data are used to compute event-related optical signals. The quasicontinuous recording is segmented into epochs time-locked to the individual stimulation (or motor responses), and the different epochs are averaged together, in a manner similar to that used for recording ERPs. Note-From "Non-Invasive NIR Optical Imaging of Human Brain Function With Sub-Second Temporal Resolution," by M. Fabiani, G. Gratton, and P. M. Corballis, 1996, Journal of Biomedical Optics, 1, p. 391. Copyright 1996 by the Society of PhotoOptical Instrumentation Engineers. Reprinted with permission.
\end{abstract}

vantages of time-resolved measures are reviewed in more detail in the Appendix (section 3). Briefly, time-resolved measures allow investigators to attain a good spatial resolution without sacrificing temporal resolution.

Two types of measures related to the passage of light through the head can be computed with a time-resolved instrument. The first is a measure of the amount of light passing through the head (i.e., intensity); the second is a measure of the time taken by photons to travel through the head (i.e., photon delay). If a frequency-domain method is used (see Appendix, section 3), this time is expressed in one of three ways: (1) as phase delay (in degrees of angle) of the signal passing through the head with respect to the input signal, or with respect to a baseline level; (2) as time delay of the signal (in picoseconds, $1 \mathrm{ps}=10^{-12} \mathrm{sec}$ ), which can be directly derived from the phase-delay measure; or (3) as a statistical transformation (e.g., $t$ scores or $z$ scores) of one of the other two measures.

Our previous studies (G. Gratton, 1997; G. Gratton, Corballis, Cho, Fabiani, \& Hood, 1995; G. Gratton, Fabiani, et al., 1997; G. Gratton, Fabiani, et al., 1995; G. Gratton, Fabiani, Goodman-Wood, \& DeSoto, 1998; for a review see Fabiani, G. Gratton, \& Corballis, 1996) show that the phase-delay measure is very sensitive to fast changes in brain activity, and, therefore, most of our work has focused on this measure (or its transforma- tions). However, both intensity and photon delay are likely to be sensitive, albeit in a different manner, to changes in scattering and absorption.

\section{Path of Light Within the Brain}

The spatial specificity of observations obtained with optical methods depends on the volume investigated with each measurement. Because the head is a scattering medium, this volume depends not only on the location of the source and of the detector but also on the optical and geometrical properties of the head itself. As already mentioned, the motion of photons through a scattering medium can be considered as a diffusion process. Thus, NIR photons traveling from a particular point (source) to another point (detector) can follow very different trajectories. This results in uncertainty about the location that is measured, quite different from what happens for $\mathrm{X}$ rays or gamma rays, which are not scattered by tissue. However, the trajectories followed by different NIR photons can be described statistically. Specifically, we can consider the probability $p$ that any particular small volume (or voxel) within the head will be traversed by a photon traveling between the source and the detector, located on the head surface. The collection of voxels with a $p$ greater than a minimum criterion can be considered as the volume investigated by a particular measurement. We will label this 


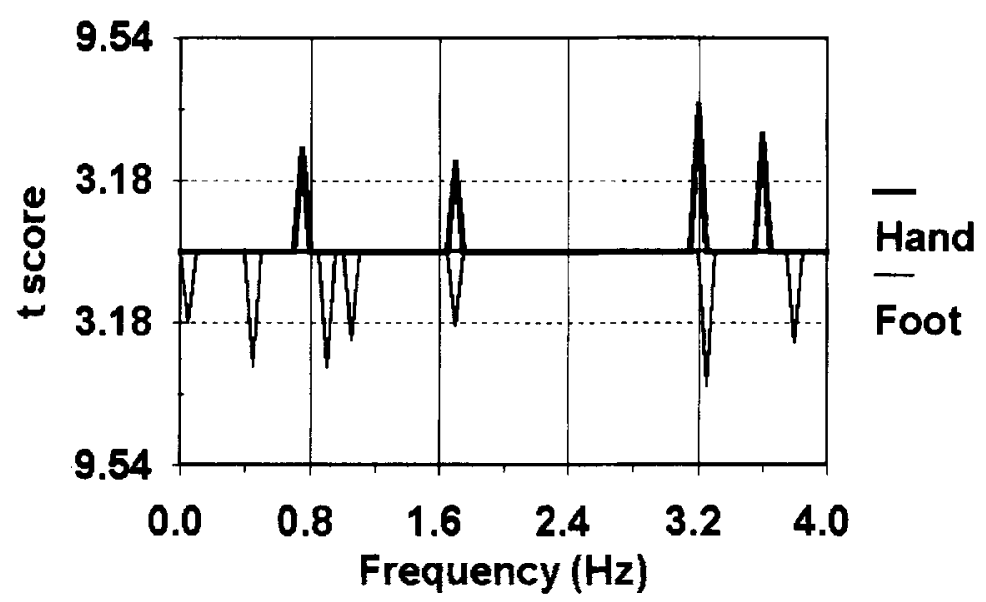

\begin{abstract}
Figure 6. Results of the tapping experiment. The figure indicates the frequency at which a significant difference was observed for recording sides contralateral and ipsilateral to the tapping limb (hand-tapping conditions are shown as upward deflections, foot-tapping conditions are shown as downward deflections). Significant effects tend to cluster around the tapping frequency $(0.8 \mathrm{~Hz})$ and some of its harmonics (1.6 and 3.2 Hz). Note-From "Rapid Changes of Optical Parameters in the Human Brain During a Tapping Task," by G. Gratton, M. Fabiani, et al., 1995, Journal of Cognitive Neuroscience, 7, p. 451. Copyright 1995 by MIT Press. Reprinted with permission.
\end{abstract}

volume the path followed by the photons between the source and the detector. Measurement values will then be considered as properties of this volume within the head, which provides the spatial specificity of the measurement. An example of this path is the crescent-shaped area shown in Figure 5.

The path followed by photons through the head is influenced by a number of factors, including the locations of source and detector, the geometry of the head, the optical properties of the tissue, and the parameters of the light sources used (i.e., wavelength and modulation frequency). Because the head (a scattering medium) is bounded by air (which is nonscattering), photons traveling between a source and a detector located on the surface of the head will tend to follow a curved path, penetrating some depth into the medium (see Figure 5). This occurs because photons traveling close to the surface of the head are likely, in their random motion, to exit from the head before reaching the detector. At that point, their movement becomes rectilinear, so that they never reenter the head and reach the detector. Only photons that travel deep within the head are likely to reach the detector. For this reason, the overall path is curved, approximating a semicircle. This counterintuitive prediction has been confirmed by Monte Carlo simulations (G. Gratton, Fabiani, et al., 1995; E. Okada, Firbank, \& Delpy, 1995; Villringer \& Chance, 1997) and demonstrations using phantoms (e.g., G. Gratton, Maier, Fabiani, Mantulin, \& E. Gratton, 1994; see Appendix, section 4).

These studies led to the conclusion that appropriate source-detector distances should allow investigators to measure optical phenomena in cortical areas using sources and detectors located on the surface of the head. A source-detector distance of about $3 \mathrm{~cm}$ is adequate to measure brain activity occurring at depths up to $3 \mathrm{~cm}$ from the head surface. In addition, when appropriate procedures are followed (see Appendix, sections 3 and 4), the width of the path of light is less than one fifth of the source-detector distance. This gives a spatial specificity of approximately $5 \mathrm{~mm}$. In conclusion, by manipulating the source-detector distance and the modulation frequency of the light source, it is possible to make these measures specific to small regions of the cortex (a few millimeters wide). Of course, appropriate modeling of the photon migration should take into account the fact that the head tissue is formed by several different layers with different optical properties (described in the Appendix, section 5).

As mentioned earlier, there is in fact evidence from animal research that scattering effects occur in active brain areas. Rector et al. (1995, 1997; see also Andrew \& MacVicar, 1994; Federico et al., 1994) observed localized changes in light scattering concurrent with cell depolarization (i.e., with neuronal activation). These findings lead to the prediction that neuronal activation in the cortex should produce changes in photon delay measurable from the surface of the head with an expected spatial localization of about $5 \mathrm{~mm}$. Experiments supporting this prediction are reviewed in the following section.

\section{EXPERIMENTS WITH EROS}

In this section, we will describe a series of experiments in which EROS was used to investigate brain activity. The purpose of this description is twofold. First, we 


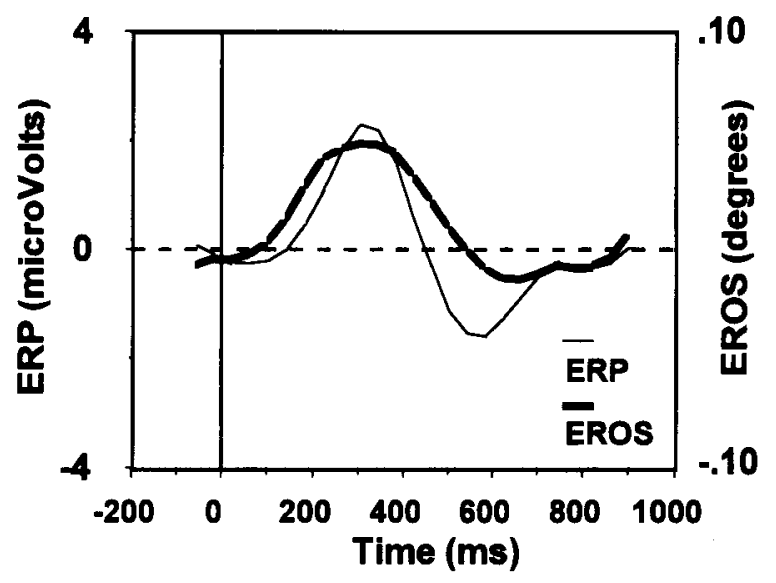

Figure 7. Time course of the EROS response (thick line) and ERP response (thin line) from one subject in a reaction-time experiment. The data are expressed as differences between locations contralateral and ipsilateral to the movement, averaged across right- and left-hand responses. The average reaction time for this subject was around $\mathbf{3 0 0} \mathbf{m s e c}$.

intend to show that EROS is a sensitive and valid method for studying the time course of activity in selected cortical areas. Second, we intend to show how EROS measures can be used to address issues that are relevant to psychologists and cognitive neuroscientists. Because most of the experiments described are already published elsewhere, their description will be schematic.

\section{EROS Related to Motor Activity}

Tapping experiment. G. Gratton, Fabiani, et al. (1995) ran an experiment to demonstrate that noninvasive optical imaging can reveal changes in brain activity that occur quite rapidly (i.e., with a frequency $>0.8 \mathrm{~Hz}$ ). Hemodynamic effects are expected to have a frequency range below $0.5 \mathrm{~Hz}$ (Rector et al., 1995). The ability of EROS to reveal activity above $0.8 \mathrm{~Hz}$ suggests that this method may be sensitive to scattering effects and, therefore, may be more closely related to neuronal activity than to the hemodynamic phenomena studied with fMRI and PET (see Malonek \& Grinvald, 1996). G. Gratton, Fabiani, et al. (1995) asked subjects to tap with one of their limbs (right foot, left foot, right hand, or left hand in different blocks) at a frequency of $0.8 \mathrm{~Hz}$, and they studied oscillations of the signal that occurred at the tapping frequency or one of its harmonics. Optical parameters were recorded from scalp locations proximal to the motor cortex over the left and right hemispheres. Differences between the recordings from locations contralateral and ipsilateral to the tapping limb were considered as indications that the signal was localized (at least to some extent) to one hemisphere and, therefore, possessed at least some degree of spatial specificity. Given the contralateral organization of the motor system, we expected the signal to be most evident over the contralateral hemisphere.
Some of the results of this experiment are presented in Figure 6 . They show that changes in the optical signal could be observed at the tapping frequency $(0.8 \mathrm{~Hz})$ and its harmonics $(1.6 \mathrm{~Hz}, 3.2 \mathrm{~Hz}$, etc.). Furthermore, for hand tapping, the signal was larger over the contralateral hemisphere, whereas, for foot tapping, a smaller degree of lateralization was observed. This may occur because the motor areas associated with the feet are located very medially, within the fold between the two hemispheres. Alternatively, foot tapping may involve more bilateral activation than hand tapping. Slow optical changes (involving effects developing over several seconds) were also observed. These signals appear to correspond to the slow NIRS signals reported by Hirth et al. (1997).

Reaction-time study. G. Gratton, Fabiani, and DeSoto (1998) investigated EROS in a task requiring speeded motor responses (RT task). One of the aims of this study was to investigate the relationship between movementrelated EROS and movement-related ERPs. The paradigm required subjects to respond with the left hand on some trials and with the right hand on other trials. This allows for the computation of the LRP, which is obtained by subtracting ERP activity measured from electrodes ipsilateral to the side of response from activity at homologous contralateral sites. In previous research (Coles, 1989; de Jong, Wierda, Mulder, \& Mulder, 1988; G. Gratton et al., 1988), it had been shown that this measure can be used to provide an "on-line" analog index of the relative preference for one or the other response during a twochoice RT task. Several pieces of evidence, including ERP source modeling (Bocker, Brunia, \& Cluitmans, 1994a, 1994b), MEG studies of movement-related potentials (Y. C. Okada, Williamson, \& Kaufman, 1982), depth electrode recordings (Arezzo, Vaughan, \& Koss, 1977), and single-unit studies (Riehle \& Requin, 1993), led to the hypothesis that the premovement LRP is generated in primary motor cortex.

G. Gratton, Fabiani, and DeSoto (1998) recorded simultaneously ERPs and EROS from a number of locations over the motor cortex and adjacent areas. Data obtained from one subject in this experiment are presented in Figures 7 and 8 . Figure 7 shows the time course of the electrical LRP and of a similar measure obtained on the basis of EROS data (the "optical LRP"). This figure indicates that the time course of the two measures is initially quite similar: Both electrical and optical LRP waveforms peak at around 300-msec latency, which was the average RT for the subject. After the movement, the electrical LRP shows a negative deflection that is not observable in the optical trace. One explanation for this difference is that the postmovement LRP may actually be sensitive to the contribution of areas related to somatosensory feedback, whereas the EROS-based LRP is not. This is consistent with current interpretations of postmovement scalp potentials (Weinberg, Cheyne, \& Crisp, 1990). Figure 8 shows the spatial distribution of the EROS activity at the time of movement with respect to the surface of the brain. The trajectory of the central sulcus derived from 


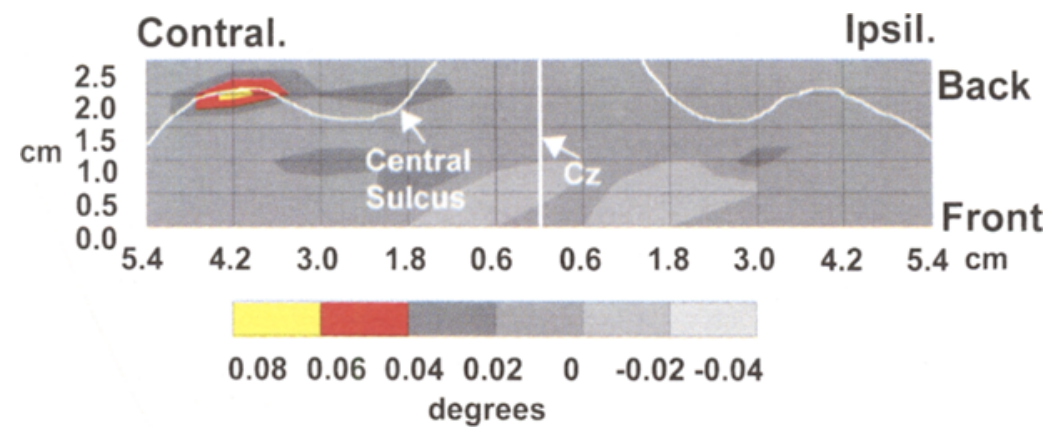

\begin{abstract}
Figure 8. Map of the average EROS response (in degrees of phase) between 200 and $400 \mathrm{msec}$ after stimulation, with respect to a prestimulus baseline level from one subject in a reaction-time experiment. EROS activity from locations contralateral to the responding hand is presented to the left, and EROS activity from locations ipsilateral to the responding hand is presented to the right, with midline locations at the center. The trajectory of the central sulcus (averaged between the left and right hemispheres) was obtained using structural MRI and traced over the corresponding optical recording locations. The scalp location of the $\mathrm{Cz}$ electrode is also reported.
\end{abstract}

structural MRI images was traced over the EROS maps. This figure shows that the focus of EROS activity is localized on the contralateral cortex just in front of the central sulcus, $4 \mathrm{~cm}$ to the side of midline. This location corresponds to the expected location of the finger section of the motor homunculus.

These data indicate that (1) EROS possesses a temporal specificity that is comparable to that of electrical measures, (2) EROS possesses a good localization power, identifying foci of activity to small cortical areas (at or below the level of 1-cm resolution), and (3) optical and electrical recordings may, in some cases, be dissociated, perhaps because of the greater spatial specificity of the optical signal, which may not be influenced by the more distant feedback areas (see also Rector et al., 1995, for similar conclusions about invasive optical recordings from the hippocampus).

\section{EROS in Occipital Areas and Relationship With fMRI and the Visual Evoked Potential (VEP)}

Grid reversal experiment. The experiments described above support the notion that EROS can be used to monitor neural activity in motor areas. We have also run several experiments investigating EROS in occipital areas in response to visual stimulation. The purpose of the first of these experiments (G. Gratton, Corballis, et al., 1995) was to demonstrate that the EROS elicited by visual stimuli could be recorded in visual areas and to investigate the time course and spatial specificity of this response. For this reason, in separate blocks, different quadrants of the visual field were stimulated every $500 \mathrm{msec}$ by the reversal of vertical black and white grids. The results indicated that the grid reversals elicited a response with a latency of approximately $100 \mathrm{msec}$ and a spatial localization that depended on the stimulated quadrant. This response was consistent with the inverted, contralateral representation of the visual field in primary visual cortex. Some of the results of this experiment are reported in Figure 9 (time course of the response) and Figure 10 (spatial resolution in the vertical and horizontal dimensions).

The same subjects were also run in similar experiments in which the VEP elicited by the grid reversals and the BOLD-fMRI activity associated with stimulation of the same areas of the visual field were studied (G. Gratton, Fabiani, et al., 1997). This allowed for a comparison of the time course of EROS with that of the VEPs and a comparison of the localization of the signal studied with EROS with that of the BOLD-fMRI activity. The results of the EROS-VEP comparison indicated that the time courses of EROS and VEPs are similar, with a high correlation across subjects and conditions. This supports the view that EROS and VEPs are both indices of neuronal activity. Note that a correlation between evoked scattering and electrophysiological changes, with latency differences within $5 \mathrm{msec}$, was reported in hippocampal slices by Rector et al. (1997). These findings are also consistent with the temporal overlap between optical and electrophysiological activity obtained in motor areas (see above).

The spatial comparison with $\mathrm{fMRI}$ indicated a close correspondence between the locus of the EROS and that of the BOLD-fMRI signals in medial occipital cortex (presumably area V1), with distances below the spatial sampling rate used for the optical measures $(0.5 \mathrm{~cm}$ on the vertical dimension and $1.5 \mathrm{~cm}$ on the horizontal dimension). In the same study, fMRI responses were also observed in more lateral occipital areas, presumably corresponding to a section of extrastriate visual cortex. Analysis of the optical response from corresponding recording locations indicated that the EROS response from these areas occurred at a longer latency with respect to the activity in striate areas (respectively, $200-300 \mathrm{msec}$ and 


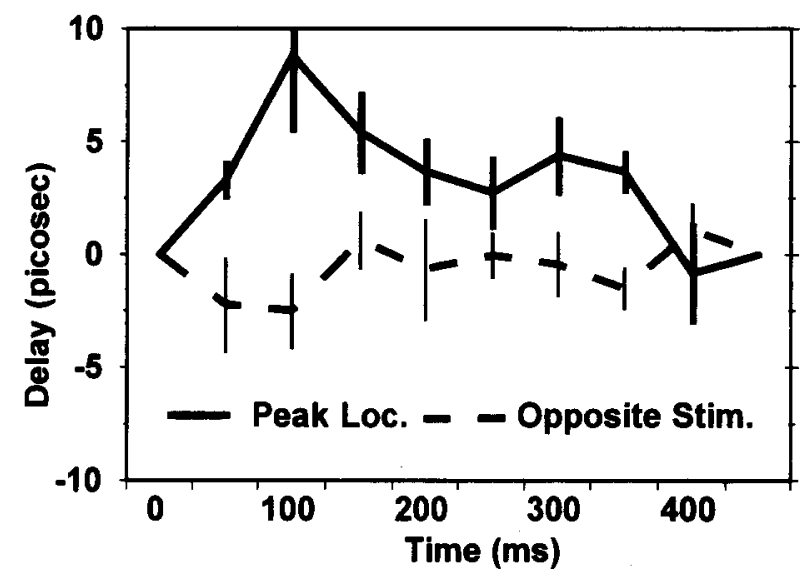

Figure 9. Time course of the EROS response in the grid reversal experiment for recording locations in which each of the grids elicited the maximum response (solid line). The response was averaged across stimulation condition and subjects. The dashed line indicates the time course of the response from the same locations when the stimulated quadrant was opposite to that producing the maximum response in that location. Note-From "Shades of Gray Matter: Noninvasive Optical Images of Human Brain Responses During Visual Stimulation," by G. Gratton, P. M. Corballis, E. Cho, M. Fabiani, and D. Hood, 1995, Psychophysiology, 32, p. 507. Copyright 1995 by Cambridge University Press. Reprinted with permission.

$100 \mathrm{msec}$; see Figure 11). This suggests that EROS can be used to determine the time course of neural activity in areas showing fMRI activity, more precisely than one can do with fMRI. ${ }^{8}$ Finally, analysis of optical activity from areas showing no fMRI response indicated that no EROS response was recorded there, suggesting that the EROS response is specific to areas showing hemodynamic signs of neuronal activation.

The results of these comparisons provide further support for the claim that EROS can be used to study the time course of neuronal activity in localized cortical areas. The temporal specificity of EROS is comparable to that of VEPs, and its spatial specificity is consistent with that of fMRI, although its spatial resolution may be more limited. A limitation of the G. Gratton, Corballis, et al. (1995) and G. Gratton, Fabiani, et al. (1997) studies is that no 3D reconstruction was used (see Appendix, section 6). Therefore, no depth information was available beyond the constraint that this method investigates an area extending to a maximum depth of $2-3 \mathrm{~cm}$ (which is a depth consistent with the fMRI data).

Spatial selective attention in primary and extrastriate cortex. G. Gratton (1997) investigated changes in EROS from occipital areas as a function of whether the eliciting stimulus was presented at an attended or unattended location. Previous studies (Clark \& Hillyard, 1996; Heinze et al., 1994) suggested that the evoked response in area Vl (primary visual cortex) is independent of attention, whereas that in extrastriate cortex is modulated by attention. In other words, activity in area VI can be considered as an "automatic" or "obligatory" response to the presentation of a stimulus (Hackley, 1993). The evidence obtained in these studies was based on dipole analysis of VEP guided by information from PET or structural MRI (Clark \& Hillyard, 1996; Heinze et al., 1994). Because dipole analysis does not provide unique solutions even when constrained by information from other brain imaging methods, this evidence cannot be considered as conclusive. In this case, EROS can be used as an independent converging method to corroborate these findings.

G. Gratton (1997) instructed subjects to maintain fixation on a cross for the duration of each block. An arrow presented at the beginning of each block indicated whether subjects had to pay attention to stimuli (squares or, more rarely, rectangles) presented to the left or to the right of the fixation cross. The subjects' task was to press a button every time a rectangle was presented on the attended side. EROS was recorded from a number of locations over the occipital cortex, mapping both $\mathrm{V} 1$ and adjacent cortical areas.

Two types of effects were investigated. The first was the effect of stimulation (i.e., the response to all stimuli regardless of whether or not they were). The second was the effect of attention, defined as the difference between the EROS elicited by stimuli presented in attended locations and the EROS elicited by the same stimuli when attention was directed to the other side of fixation. Maps of these effects within the interval extending between 40 and $140 \mathrm{msec}$ after stimulation (corresponding to the latency of the VEP effects reported by Heinze et al., 1994) are reported in Figure 12.

These maps indicate that the stimulation effect is maximum in a medial area (presumably corresponding to area V1), whereas the attention effect is maximum in a more lateral area (presumably corresponding to extrastriate cortex). No significant attention effect was observed in medial occipital areas. This supports the hypothesis that the response in primary visual cortex (V1) is independent of attention and that attention effects begin to take effect in subsequent stations of the visual pathway (Hackley, 1993).

Memory-driven processing in V1. In the experiment reported by G. Gratton (1997), it was also possible to examine the difference in the EROS elicited by the rare stimuli (the rectangles) and the frequent stimuli (the squares). This comparison revealed that the EROS response in V1 was larger for rare stimuli than for frequent stimuli. Differences in the amplitude of the responses elicited by rare and frequent stimuli are commonly found for many ERP components (beginning at least with the auditory N1; Lu, Williamson, \& Kaufman, 1992). These effects are often interpreted as due to sensory or other memory phenomena, which bring forth an attenuation of the response elicited by frequently repeated stimuli (Ritter, Deacon, Gomes, Javitt, \& Vaughan, 1995). However, within the visual domain, phenomena of this type have been reported only for components with a much longer latency $(>150 \mathrm{msec})$. In addition, the dominant view of 
A

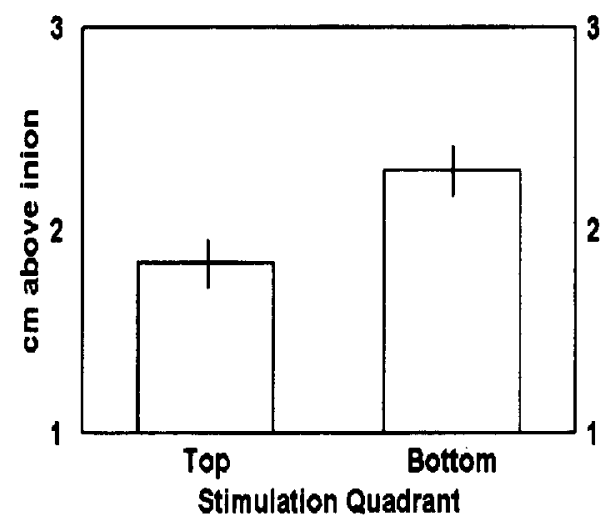

B

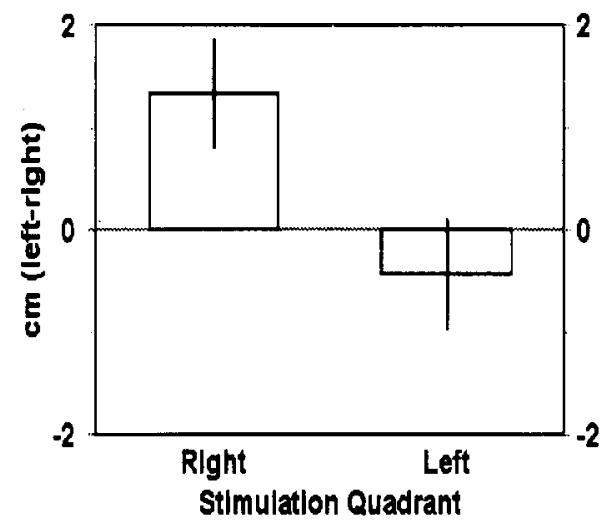

Figure 10. Results of the grid stimulation experiment. Panels $A$ and B show the localization power of EROS in the vertical dimension and in the horizontal dimension, respectively (latency of the EROS response $=$ $100 \mathrm{msec}$ ). The coordinates are expressed as distances from the inion. On average, primary visual cortex can be expected to lay close to the midline, $2-2.5 \mathrm{~cm}$ above the inion. In agreement with the typical contralateral inverted representation of the visual field in area $\mathrm{V} 1$, stimulation of the bottom quadrants of the visual field results in activation of the top banks of the primary visual cortex, and vice versa (panel $A$ ). In addition, stimulation of the right or left hemifield results in activation of the visual cortex in the hemisphere contralateral to stimulation (panel B). Note that the spatial sampling of EROS data was greater for the vertical dimension than for the horizontal dimension and, thus, accounts for the smaller error bars for the vertical discrimination.

the activity in V1 emphasizes the stereotyped, data-driven nature of the processing occurring in this area (e.g., Hubel $\&$ Wiesel, 1979). This view is reinforced by the observation that activity in this area appears to be outside of the control of attention (see above).

The probability effects reported by G. Gratton (1997) may suggest a more flexible view of Vl activity. However, it should be considered that, in this experiment, the probability effect was confounded with stimulus characteristics (the rare stimulus was a rectangle and the frequent stimulus a square) and response requirements. In addition, the same two retinal positions were used for all stimuli, making it possible for peripheral receptor habituation to account for some of the findings. This left open the question of whether the activity in Vl could be influenced by memory processes.

Therefore, G. Gratton, Fabiani, et al. (1998) ran another study in which EROS was recorded during a modified Sternberg's memory-search paradigm (Sternberg, 1966; see Figure 13). This paradigm was based on previous results (see G. Gratton, Corballis, \& Jain, 1997) indicating that visual memory is, at least in part, hemispherically organized. That is to say, visual stimuli presented to the left and right of fixation leave memory traces that are stronger in the hemisphere contralateral with respect to the side of stimulation. As shown in Figure 13, subjects were instructed to fixate on a cross presented at the center of a computer display. Each trial began with the presentation of the memory set, comprising two letters flashed to the left and right of the fixation cross. After $2 \mathrm{sec}$, a new letter (test stimulus) was flashed just above the fixation cross. The subject's task was to determine whether the test letter belonged to the memory set. EROS was recorded from occipital areas encompassing V1 and adjacent cortical regions in the two hemispheres.

The focus of the analysis was on the EROS elicited by test stimuli. Three conditions were identified: The test letter could be the same as the memory set letter presented to the left ("OLD LEFT" condition), the same as the one presented to the right ("OLD RIGHT" condition), or an entirely new letter ("NEW" condition). The crucial comparison was between the EROS elicited by the OLD LEFT and OLD RIGHT conditions from the left and right primary visual cortices. Note that the stimuli eliciting these EROS responses were matched for stimulus type and response requirements. Furthermore, a different retinal position was used for the study and test phases of the trial. We hypothesized that the memory set stimuli would leave a stronger memory trace in the hemisphere contralateral to the side of stimulation. We hypothesized that the activity in V1 is affected (or "driven") by memory processes. If these hypotheses are true, the EROS in the left and right Vl areas should differ depending on whether the test stimulus matched the memory set stimulus presented to the left (OLD LEFT condition) or the memory set stimulus presented to the right (OLD RIGHT condition). ${ }^{9}$ In particular, given the results obtained by G. Gratton (1997), it was predicted that the response would be smaller in the hemisphere previously exposed to the stimulus (i.e., right hemisphere for the OLD LEFT condition and left hemisphere for the OLD RIGHT condition) than in the opposite hemisphere.

Maps of the initial EROS response (latency between 40 and $140 \mathrm{msec}$ ) elicited by OLD LEFT, OLD RIGHT, and 


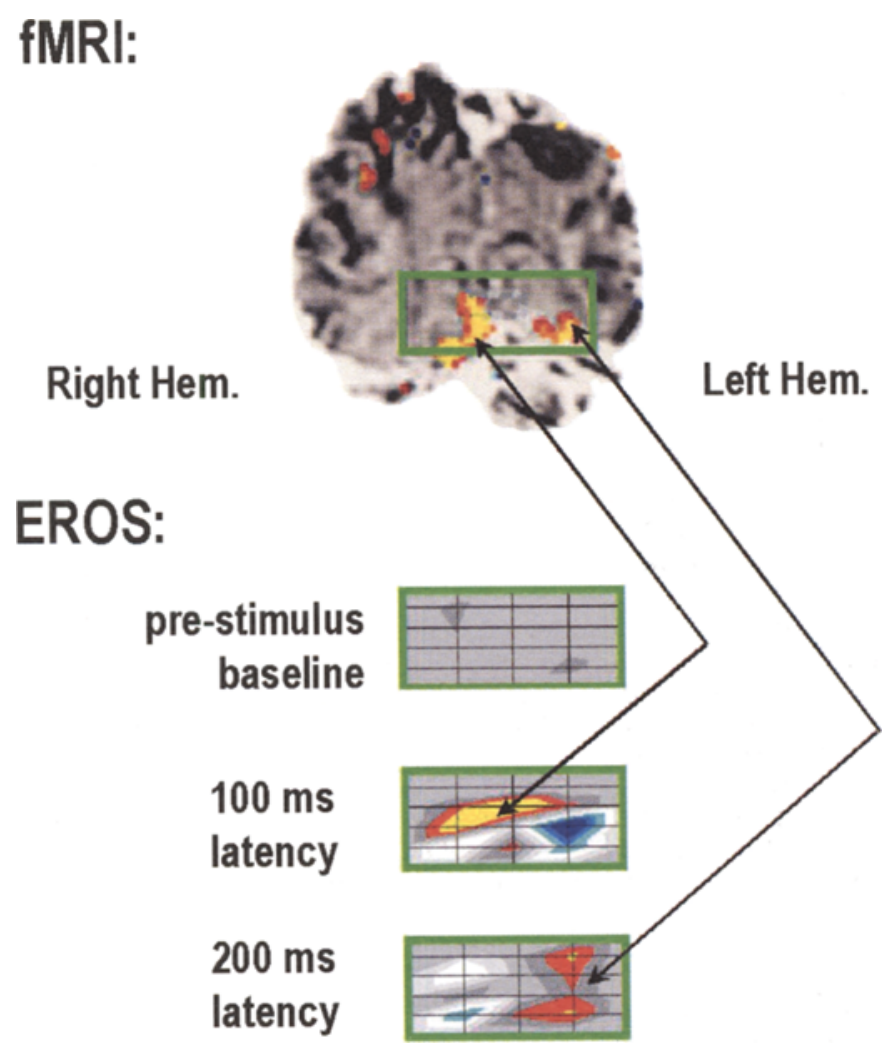

Figure 11. Comparison of $\mathrm{AMRI}$ and EROS responses in the grid stimulation experiment. The data refer to stimulation of the upper left quadrant of the visual field in one subject. The upper panel reports the BOLD-fMRI slice with maximum activity in this condition. Voxels in yellow are those exhibiting the most significant BOLD response. The rectangle shown in green corresponds to the area explored with EROS. Maps of the EROS response before stimulation and 100 and $200 \mathrm{msec}$ after grid reversal are shown at the bottom of the figure, with areas in yellow indicating locations of maximum activity. Note that the location of maximum EROS response varies with latency: The earlier response $(100 \mathrm{msec}$ ) corresponds in location to the more medial fMRI response, whereas the later response $(200 \mathrm{msec})$ corresponds in location to the more lateral $f$ MRI response. The data are presented following the radiological convention (i.e., the brain is represented as if it were seen from the front).

NEW stimuli are presented in Figure 14. These maps show that the EROS response was attenuated in the hemisphere previously exposed to the test stimulus with respect to the response in the opposite hemisphere. The contralateral response was also attenuated with respect to the response obtained with new stimuli. This finding indicates that activity in primary visual cortex is influenced by memory processes. This can be accounted for by either of two mechanisms: (1) a memory trace that persists in medial occipital cortex between the presentation of the memory set and that of the test stimulus or (2) a patternrecognition mechanism set up in this area as a function of memory traces maintained or activated in a different area (e.g., in temporal cortex). In either case, the mechanism is hemispheric specific, because differential responses were observed in the two hemispheres. ${ }^{10}$
These results invite a revision of the traditional notion that activity in V1 is stereotyped and data-driven and suggest instead the operation of hemispheric-specific, rapidlyadapting, memory-driven processing in this area. Both the spatial selective attention experiment and the experiment on memory-driven processing in medial occipital cortex demonstrate the power of methods that provide both time- and location-specific information.

\section{DISCUSSION}

The main purposes of this paper were to describe the concept of dynamic brain imaging and to introduce a new methodology, EROS, which is particularly useful for this purpose. We view dynamic brain imaging as one of several tools (including single-unit recordings, lesion data, etc.) 


\title{
Contralateral vs. Ipsilateral Effects Lat: $\mathbf{4 0 - 1 4 0 ~ m s}$ \\ Stimulation \\ Attention
}

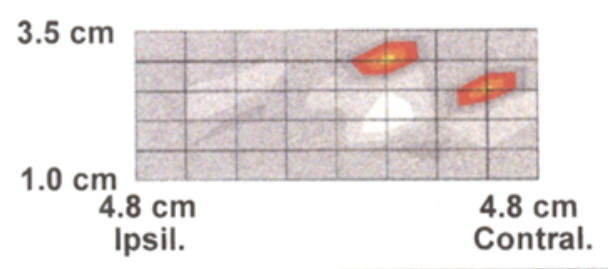

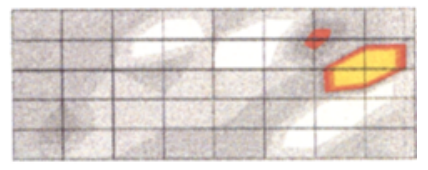

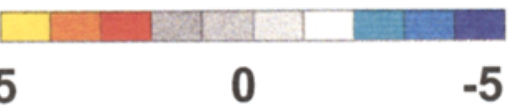

Phase (t score)

\begin{abstract}
Figure 12. Results of the attention experiment. $t$-score maps (computed across subjects) of the stimulation (left map) and attention (right map) EROS effects at a latency between 40 and $140 \mathrm{msec}$ after stimulation. The coordinates of the maps are expressed relative to the inion. Areas in yellow indicate locations of maximum response. Both medial and lateral visual areas are activated as a result of stimulation. However, attention effects are visible only in lateral visual areas. Note-From "Attention and Probability Effects in the Human Occipital Cortex: An Optical Imaging Study,” by G. Gratton, 1997, NeuroReport, 8, p. 1751). Copyright 1997 by Lippincott Williams \& Wilkins. Reprinted with permission.
\end{abstract}

that can provide data about how neural activity evolves over time within or across particular regions of the brain. This information can then be used to formulate, refine, and test theories about how the mind/brain processes information. For instance, the data obtained by G. Gratton, Fabiani, et al. (1998) could be used to refine theories about the processing of visual information. In particular, they indicate that top-down, memory-driven processes may have an impact even on the earliest stages of information processing. Furthermore, the combination of these data with those obtained by G. Gratton (1997) may indicate that these memory-driven processes occur at a level in which attention does not play a role. Similar ideas were also developed on the basis of other measures of brain activity, such as the mismatch negativity, an ERP phenomenon indicating sensory memory activity in early stations of the auditory systems that are not affected by attention (Näätänen, 1992; Ritter et al., 1995).

A crucial aspect of the elaboration of a dynamic brain imaging approach is the development of procedures that allow investigators to make statements about brain activity that have both spatial and temporal specificity. As described earlier, different techniques vary in the type of spatial and temporal information they provide, with electrophysiological techniques excelling in the temporal domain, and hemodynamic techniques excelling in the spatial domain. EROS can provide a good combination of spatial and temporal information. Other techniques may also provide a useful combination of spatial and temporal resolution, appropriate to address particular ex- perimental issues. For example, MEG is a powerful method for the investigation of sensory processes (e.g., Hari \& Lounasmaa, 1989; Lu et al., 1992; Tiitinen et al., 1993), dipole analysis of ERPs has provided useful insights about the effects of attention (e.g., Clark \& Hillyard, 1996), and the development of "single-trial" fMRI procedures (e.g., Buckner et al., 1996) has shown that hemodynamic methods may also provide some degree of trial-level temporal specificity. In addition, ERP research has for a long time used scalp distribution of the data to differentiate between neuroanatomically distinct subsets of activities (or components). Although conclusions about the brain generators of ERPs may be somewhat tentative, ERPs can provide a dynamic view of the interactions among different brain phenomena that can be very useful in experimental psychology, cognitive neuroscience, and related areas (e.g., Fabiani et al., in press; Rugg \& Coles, 1995). Thus, dynamic brain imaging should be viewed not as the exclusive realm of a particular technique but as an approach in which several different methods may provide complementary contributions, specializing in different levels of description.

Whereas our focus has so far been on the information that can be obtained with individual imaging techniques used in isolation, it is clear that different methods can be combined to provide a more complete and accurate description of brain dynamics. There are several approaches to the problem of combining procedures to obtain optimal spatial and temporal resolution. For example, the combination of electrophysiological and hemodynamic imag- 


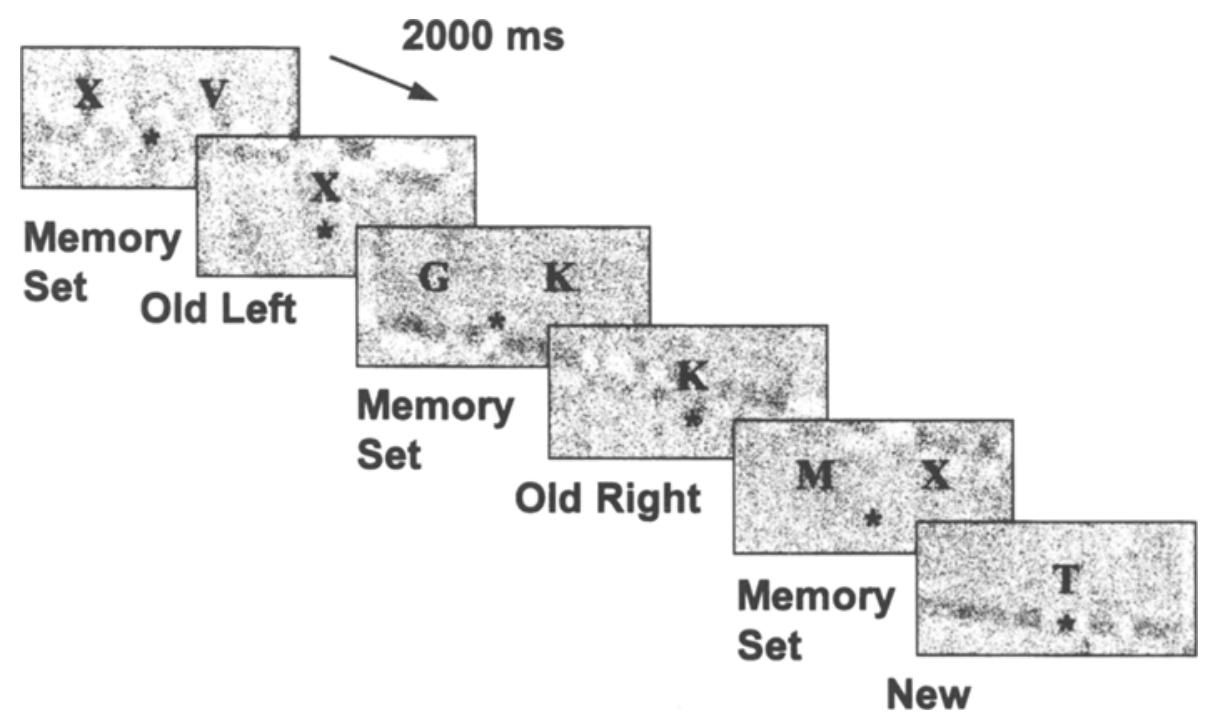

Figure 13. Schematic representation of the paradigm used in the memory experiment.

ing data has been advocated as one of the most promising approaches, and it may represent a viable solution in a number of cases (see Barinaga, 1997). This strategy involves measuring hemodynamic and electrophysiological effects in similar paradigms. The areas of the brain showing hemodynamic activity are identified. These areas are then used to constrain source models of ERP or MEG activity, which, in turn, should allow investigators to reconstruct the moment-by-moment contribution of different electrophysiological sources to the observed scalp activity and to derive spatiotemporal descriptions of the brain activity involved in a particular task.

This procedure is based on several assumptions. A fundamental assumption is that the sets of localized activities responsible for the electrophysiological and hemodynamic signals overlap. This assumption may be valid in a variety of cases. However, there are several caveats that should be considered before advocating its general use. First, a definition of the sets of activities contributing to both the hemodynamic and the electrophysiological signal may be difficult because determination of the areas that are active (with respect to those that are not) is usually based on statistical rather than absolute criteria. For example, a brain area is said to show fMRI activation if the BOLD signal in that area is significant at a particular level with respect to a baseline activation. Such a definition depends on the power (i.e., the signal-to-noise ratio and the number of observations) of a particular study, and, therefore, similar manipulations may yield different subsets of activities in different experiments. In addition, the power of electrophysiological and hemodynamic measures may differ.

Second, some brain areas may show electrophysiological activity but not hemodynamic activity, or vice versa. For instance, for electrophysiological activity to be observable at the surface of the head, neurons must possess an "open-field" configuration (i.e., they have to be aligned so that their individual field can summate; $\mathrm{Al}-$ lison et al., 1986; Lorente de Nó, 1947; Nunez, 1981). In the case of MEG signals, the electric current generated by the neurons must also be tangential to the scalp. This neuronal architecture is not required for the hemodynamic effects to be visible. In addition, hemodynamic effects may emphasize the contributions of areas that are active over extended periods of time, whereas electrophysiological measures may emphasize the contributions of areas whose level of activity changes rapidly. Finally, electrophysiological measures may emphasize the contribution of superficial as opposed to deep structures, whereas hemodynamic measures may, in some cases, be especially sensitive to phenomena occurring in the proximity of either large or small blood vessels (e.g., the "brain or vein" issue; Frahm, Merboldt, Hänicke, Kleinschmidt, \& Boecker, 1994; Villringer \& Dirnagl, 1995).

Third, depth electrode studies in humans and animals have shown that it is often possible to distinguish several electrophysiological activities with different orientation, time course, and functional significance within a cortical area just a few millimeters wide (Javitt, Schroeder, Steinschneider, Arezzo, \& Vaughan, 1992; Javitt, Steinschneider, Schroeder, Vaughan, \& Arezzo, 1994; McCarthy \& Wood, 1987). It is not clear whether hemodynamic measures could resolve these overlapping activities into different sources, since high spatial localization power does not necessarily translate into millimeter-level spatial resolution (i.e., into the ability to discriminate active areas located a few millimeters apart). In fact, two sources may be too large and/or too close to each other to be resolved effectively. If hemodynamic imaging data are used to constrain the number of areas that are active 


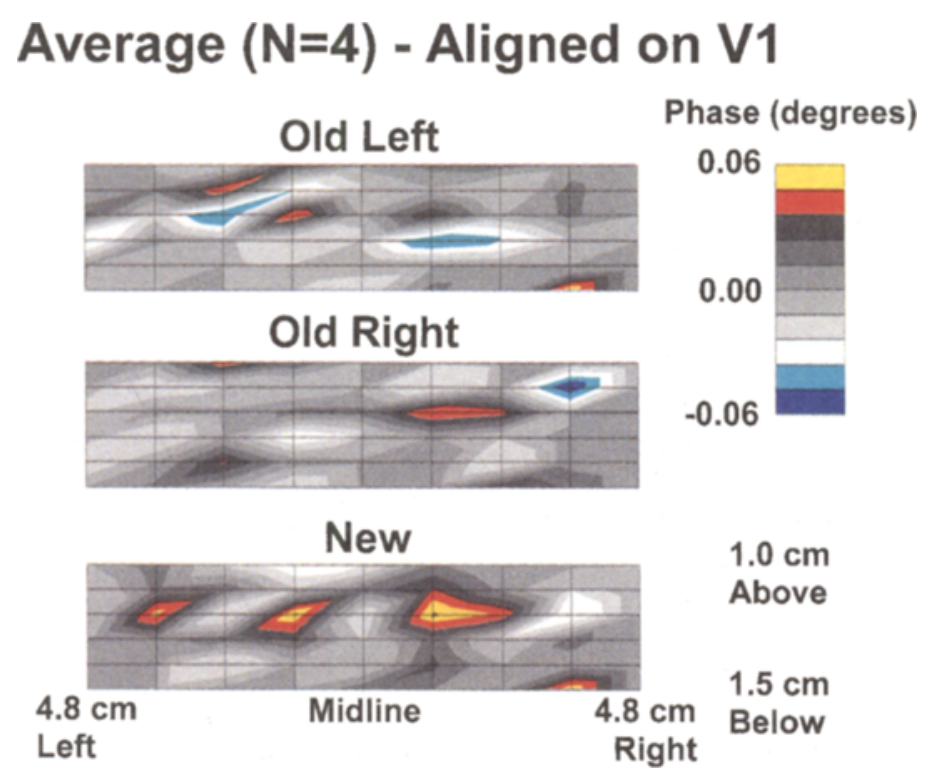

Figure 14. Maps of the EROS response between a latency of 40-140 msec after stimulation (computed with respect to a prestimulus baseline) for the OLD LEFT (top panel), OLD RIGHT (middle panel), and NEW (bottom panel) conditions in the memory experiment. The maps were aligned on the location of maximum response across conditions (presumably V1) and averaged across subjects. The coordinates are expressed relative to the midpoint between the expected location of $V 1$ in the left and right hemispheres. Areas in yellow indicate locations of maximum response. Note-From "Memory-Driven Processing in Human Medial Occipital Cortex: An Event-Related Optical Signal (EROS) Study," by G. Gratton, M. Fabiani, M. R. Goodman-Wood, and M. C. DeSoto, 1998, Psychophysiology, 35, p. 350 . Copyright 1998 by Cambridge University Press. Reprinted with permission.

during a particular task, uncertainty would remain on how many electrophysiological sources to place within each area of activation. Thus, although a combination of hemodynamic brain imaging data and electrophysiological measures may provide useful spatiotemporal descriptions of brain activity (as in the examples given above), this approach requires support from converging evidence and some degree of external validation.

Fourth, hemodynamic measures may be sensitive only to overall changes in neural activity, but not to changes in the temporal pattern of neuronal firing (e.g., frequency modulation and spike synchronization; Riehle, Gruen, Diesmann, \& Aertsen, 1997).

Notwithstanding these difficulties, the combination of different methodologies may still provide researchers with a very powerful tool. Some of the problems in combining methods can be reduced by recording the different measures simultaneously. In addition, the spatial and temporal specificity of the different measures should be made as close and congruent as possible. For instance, fMRI, PET, and EROS data are best compared with source analysis of ERP data rather than with surface maps (since the latter represent the summation of activity from different areas; G. Gratton, Fabiani, et al., 1997; Heinze et al., 1994). Optical measures may play a useful role, because of their recording compatibility with other measures, because of their spatial and temporal resolution, and because of the possibility of simultaneously recording fast phenomena (related to neuronal activity) as well as slow phenomena (related to hemodynamic events). However, a significant limitation of optical methods appears to be their limited penetration, which may make it difficult to use them to image brain structures that are relatively distant from the surface of the head and are surrounded by white matter. In the future, it may be possible to image structures that are close to the base of the skull by placing source and/or detector fibers in some head cavity (such as the mouth or the nose).

In conclusion, in this paper, we presented an approach to the study of the brain that focuses on the dynamic interactions among different areas. This approach corresponds to emerging views of the mind/brain that emphasize that most cognitive functions are the result of activity across complex neural networks involving a number of different brain areas and mental processes (e.g., Mesulam, 1990; Squire, 1989). In this paper, we have reviewed the use of noninvasive imaging methods, focusing on their ability to provide information with both spatial and tem- 
poral specificity. In particular, we have described a methodology, EROS, which combines both types of information and can be used to study the time course of activity in specified cortical areas. Other advantages of EROS include (1) low cost, (2) portability, (3) noninvasiveness, (4) compatibility with other methods, and (5) good spatial localization, without the need to rely on modeling (unlike ERPs and MEG). The major restriction to the use of EROS is its limited penetration. We have then discussed the combined use of different techniques, as well as the advantages and limitations that can be expected in this process. Integration of functional imaging data with behavioral cognitive data and with data obtained with more invasive methods such as single-unit and far-field recordings (both in humans and animals), lesion studies, pharmacological studies, and so forth can further refine our views about the mind and the brain.

\section{REFERENCES}

Alfano, R. R., Demos, S. G., \& Gayen, S. K. (1997). Advances in optical imaging of biomedical media. In D. S. Lester, C. C. Felder, \& E. N. Lewis (Eds.), Imaging brain structure and function: Emerging technologies in the neurosciences (Annals of the New York Academy of Sciences, Vol. 820, pp. 248-270). New York: New York Academy of Sciences.

Allison, T., Wood, C. C., \& McCarthy, G. (1986). The central nervous system. In M. G. H. Coles, S. W. Porges, \& E. Donchin (Eds.), Psychophysiology: Systems, processes, and applications (pp. 5-25) New York: Guilford.

ANDREW, R. D., \& MACVICAR, B. A. (1994). Imaging cell volume changes and neuronal excitation in the hippocampal slice. Neuroscience, 62, 371-383.

arezzo, J., Vaughan, H. G., JR., \& Koss, B. (1977). Relationship of neuronal activity to gross movement-related potentials in monkey pre- and postcentral cortex. Brain Research, 132, 362-369.

ARRIDGE, S. R., \& HeBdEN, J. C. (1997). Optical imaging in medicine: II. Modeling and reconstruction. Physics in Medicine \& Biology, 42, 841-853.

ARRIDGE, S. R., \& SCHWEIGER, M. (1997). Image reconstruction in optical tomography. Philosophical Transactions of the Royal Society of London: Series B, 352, 717-726.

Bandettini, P. A., Wong, E. C., Hinks, R. S., Tikofsky, R. S., \& HYDE, J. S. (1992). Time course EPI of human brain function during task activation. Magnetic Resonance in Medicine, 25, 390-397.

BARINAGA, M. (1997). New imaging methods provide a better view into the brain [news]. Science, 276, 1974-1976.

Belliveau, J. W., Kennedy, D. N., JR., McKinstry, R. C., BuchBinder, B. R., WeissKoff, R. M., Cohen, M. S., Vevea, J. M., Brady, T. J., \& Rosen, B. R. (1991). Functional mapping of the human visual cortex by magnetic resonance imaging. Science, $\mathbf{2 5 4}$, 716-719.

Benaron, D. A., Gwiazdowski, S., Kurth, C. D., Steven, J., Delivoria-Papadopoulos, M., \& Chance, B. (1990). Optical path length of $754 \mathrm{~nm}$ and $816 \mathrm{~nm}$ light emitted into the head of infants. Annual International Conference of IEEE, 12, 1117-1119.

Benaron, D. A., Ho, D. C., Spilman, S., Van Houten, J. P., \& Stevenson, D. K. (1994). Non-recursive linear algorithms for optical imaging in diffusive media. Advances in Experimental Medicine \& Biology, 361, 215-222.

BENARON, D. A., \& Villringer, A. (1998). Noninvasive functional imaging of human brain using light. Manuscript in preparation.

Bocker, K. B., Brunia, C. H., \& Cluitmans, P. J. (1994a). A spatiotemporal dipole model of the readiness potential in humans. I. Finger movement. Electroencephalography \& Clinical Neurophysiologv, 91, 275-285.
Bocker, K. B., Brunia, C. H., \& Cluitmans, P. J. (1994b). A spatio temporal dipole model of the readiness potential in humans. II. Foo movement. Electroencephalography \& Clinical Neurophysiology 91, 286-294.

BonHOEFFER, T., \& GRINVALD, A. (1993). Optical imaging of the func tional architecture in cat visual cortex: The layout of direction anc orientation domains. Advances in Experimental Medicine \& Biology 333, 57-69.

BonhoefFer, T., \& Grinvald, A. (1996). Optical imaging based on in trinsic signals. In A. W. Toga \& J. C. Mazziotta (Eds.), Brain map. ping: The methods (pp. 55-97). San Diego, CA: Academic Press.

Broca, P. (1865). Sur le siège de la faculté du langage articulé [On the localization of the faculty of articulated language]. Bulletin of the So ciety of Anthropology, 6, 377-396.

BUCKNER, R. L. (1996). Beyond HERA: Contributions of specific pre. frontal brain areas to long-term memory retrieval. Psychonomic Bul. letin \& Review, 3, 149-158.

Buckner, R. L., Bandettini, P. A., O'Craven, K. M., Savoy, R. L. Petersen, S. E., Raichle, M. E., \& Rosen, B. R. (1996). Detectior of cortical activation during averaged single trials of a cognitive task using functional magnetic resonance imaging. Proceedings of the National Academy of Sciences, 93, 14878-14883.

Cannestra, A. F., Blood, A. J., Black, K. L., \& Toga, A. W. (1996). The evolution of optical signals in human and rodent cortex. Neurolmage 3, 202-208.

Chance, B., Kang, K., He, L., Weng, J., \& Sevick, E. (1993). Highly sensitive object location in tissue models with linear in-phase anc anti-phase multi-element optical arrays in one and two dimensions Proceedings of the National Academy of Sciences, 90, 3423-3427 [Published erratum appears in Proceedings of the National Academ] of Sciences, 1995, 92, 4074.]

Chance, B., Luo, Q., Nioka, S., Alsop, D. C., \& Detre, J. A. (1997) Optical investigations of physiology: A study of intrinsic and extrinsic biomedical contrast. Philosophical Transactions of the Royal Society of London: Series $B, 352,707-716$.

Chang, J., Graber, H. L., \& Barbour, R. L. (1997). Luminescence optical tomography of dense scattering media. Journal of the Optica. Society of America A, 14, 288-299.

Chang, J., Graber, H. L., Koo, P. C., Aronson, R., Barbour, S. L., \& BARBOUR, R. L. (1997). Optical imaging of anatomical maps derived from magnetic resonance images using time-independent optical sources: IEEE Transactions on Medical Imaging, 16, 68-77.

Churchland, P. S., \& Sejnowski, T. J. (1988). Perspectives in cognitive neuroscience. Science, 242, 741-745.

Clark, V. P., \& Hillyard, S. A. (1996). Spatial selective attention affects early extra-striate but not striate components of the visual evoked potential. Journal of Cognitive Neuroscience, 8, 387-402.

Cohen, J. D., Perlstein, W. M., Braver, T. S., Nystrom, L. E., Noll, D. C., Jonides, J., \& SMith, E. E. (1997). Temporal dynamics of brain activation during a working memory task. Nature, 386, 604-608.

COHEN, L. B. (1972). Changes in neuron structure during action potential propogation and synaptic transmission. Physiological Review, $\mathbf{5 3}$, 373-417.

Coles, M. G. (1989). Modern mind-brain reading: Psychophysiology, physiology, and cognition. Psychophysiology, 26, 251-269.

Cooper, C. E., Elwell, C. E., Meek, J. H., Matcher, S. J., Wyatt, J. S., COPE, M., \& Delpy, D. T. (1996). The noninvasive measurement of absolute cerebral deoxy-hemoglobin concentration and mean optical path length in the neonatal brain by second derivative near infrared spectroscopy. Pediatric Research, 39, 32-38.

COPE, M., \& DELPY, D. T. (1988). System for long-term measurement of cerebral blood and tissue oxygenation of newborn infants by near infra-red transillumination. Medical \& Biological Engineering \& Computing, 26, 289-294.

Corbetta, M., Shulman, G. L., Miezin, F. M., \& Petersen, S. E. (1995). Superior parietal cortex activation during spatial attention shifts and visual feature conjunction. Science, 270, 802-805.

Courtney, S. M., Ungerleider, L. G., Keil, K., \& Haxby, J. V. (1997). Transient and sustained activity in a distributed neural system for human working memory. Nature, 386, 608-611. 
DALE, A. M., \& BUCKNER, R. L. (1997). Selective averaging of rapidly presented individual trials using fMRI. Human Brain Mapping, 5 , 329-340.

de Jong, R., Wierda, M., Mulder, G., \& Mulder, L. J. (1988). Use of partial stimulus information in response processing, Journal of Experimental Psychology: Human Perception \& Performance, 14, 682 692.

DeYoe, E. A., Carman, G. J., Bandettini, P., Glickman, S., WieSER, J., CoX, R., MilleR, D., \& NeITZ, J. (1996). Mapping striate and extrastriate visual areas in human cerebral cortex. Proceedings of the National Academy of Sciences, 93, 2382-2386.

Engel, S. A., Rumelhart, D. E., Wandell, B. A., Lee, A. T., Glover, G. H., Chichilnisky, E. J., \& Shadlen, M. N. (1994). fMRI of human visual cortex [letter]. Nature, 369, 525. [Published erratum appears in Nature, 1994, 370, 106.]

ERNST, T., \& HeNNIG, J. (1994). Observation of a fast response in functional MR. Magnetic Resonance in Medicine, 32, 146-149.

Fabiani, M., Gratton, G., \& Coles, M. G. H. (in press). Event-related brain potentials. In J. Cacioppo, L. Tassinary, \& G. Berntson (Eds.), Handbook of psychophysiology. Cambridge: Cambridge University Press.

Fabiani, M., Gratton, G., \& Corballis, P. M. (1996). Non-invasive NIR optical imaging of human brain function with sub-second temporal resolution. Journal of Biomedical Optics, 1, 387-398.

Federico, P., Borg, S. G., Salkauskus, A. G., \& MacVicar, B. A. (1994). Mapping patterns of neuronal activity and seizure propagation by imaging intrinsic optical signals in the isolated whole brain of the guinea-pig. Neuroscience, 58, 461-480.

Fiez, J. A., Raife, E. A., Schwarz, J., Balota, D. A., Raichle, M. E., \& Petersen, S. E. (1996). Functional anatomical studies of the shortterm maintenance of verbal information. Journal of Neuroscience, 16, 808-822.

Fox, P. T., \& RAICHLE, M. E. (1986). Focal physiological uncoupling of cerebral blood flow and oxidative metabolism during somatosensory stimulation in human subjects. Proceedings of the National Academy of Sciences, 83, $1140-1144$

Frahm, J., Bruhn, H., Merboldt, K. D., \& Hänicke, W. (1992). Dynamic MR imaging of human brain oxygenation during rest and photic stimulation. Journal of Magnetic Resonance Imaging, 2, 501-505.

Frahm, J., Merboldt, K. D., Hänicke, W., Kleinschmidt, A., \& BOECKER, H. (1994). Brain or vein-Oxygenation or flow? On signal physiology in functional MRI of human brain activation. NMR in Biomedicine, 7, 45-53.

FrostiG, R. D. (1994). What does in vivo optical imaging tell us about the primary visual cortex in primates? In A. Peters \& K. S. Rockland (Eds.), Cerebral cortex (Vol. 10, pp. 331-358). New York: Plenum.

Frostig, R. D., Lieke, E. E., Ts'o, D. Y., \& Grinvald, A. (1990). Cortical functional architecture and local coupling between neuronal activity and the microcirculation revealed by in vivo high-resolution optical imaging of intrinsic signals. Proceedings of the National Academy of Sciences, 87, 6082-6086.

GARDNER, H. (1985). The mind's new science: A history of the cognitive revolution. New York: BasicBooks

GazZANiga, M. S. (ED.) (1995). The cognitive neurosciences. Cambridge, MA: MIT Press.

GazZaniga, M. S., Ivry, R., \& Mangun, G. R. (1998). Cognitive neuroscience: The biology of the mind. New York: Norton.

GEVINS, A. (1996). Electrophysiological imaging of brain function. In A. W. Toga \& J. C. Mazziotta (Eds.), Brain mapping: The methods (pp. 259-276). San Diego, CA: Academic Press.

Gratton, E., Gratton, G., Fabiani, M., \& Corballis, P. M. (1996). Detection of brain activity using time-resolved near infrared techniques. In Proceedings of the Fifth International Conference: Peace Through Mind-Brain Science (pp. 214-223).

Gratton, E., Jameson, D. M., Rosato, N., \& Weber, G. (1984). Multifrequency cross-correlation phase fluorometer using synchrotron radiation. Review's of Scientific Instrumentation, 55, 486-494.

Gratton, E., \& LimkEMAN, M. (1983). A continuously variable frequency cross-correlation phase fluorometer with picosecond resolution. Biophwsical Journal. 44, 315-324.

GratToN, G. (1997). Attention and probability effects in the human occipital cortex: An optical imaging study. NeuroReport, 8, 1749-1753.
Gratton, G., Coles, M. G., Sirevaag, E. J., Eriksen, C. W., \& DonCHIN, E. (1988). Pre- and poststimulus activation of response channels: A psychophysiological analysis. Journal of Experimental Psychology: Human Perception \& Performance, 14, 331-344.

Gratton, G., Corballis, P. M., Cho, E., Fabiani, M., \& Hood, D. (1995). Shades of gray matter: Noninvasive optical images of human brain responses during visual stimulation. Psychophysiology, 32 , 505-509.

Gratton, G., Corballis, P. M., \& Jain, S. (1997). Hemispheric organization of visual memories. Journal of Cognitive Neuroscience, 9 , 92-104.

Gratton, G., Fabiani, M., Corballis, P. M., Hood, D., Goodman, M. R., Hirsch, J., Kim, K., Friedman, D., \& Gratton, E. (1997). Non-invasive optical measures of localized neuronal activity in the human occipital cortex. Neurolmage, 6, 168-180.

Gratton, G., Fabiani, M., \& DeSoto, M. C. (1998). The time course of response-related brain activity in a choice reaction time task: Comparison of optical and electrophysiological measures. Neurolmage. 7, S688.

Gratton, G., Fabiani, M., Friedman, D., Franceschini, M. A., FanTINI, S., \& GRatTon, E. (1995). Rapid changes of optical parameters in the human brain during a tapping task. Journal of Cognitive Neuroscience, 7, 446-456.

Gratton, G., Fabiani, M., Goodman-Wood, M. R., \& DeSoto, M. C. (1998). Memory-driven processing in human medial occipital cortex: An event-related optical signal (EROS) study. Psychophysiology, 35, 348-351

Gratton, G., Maier, J. S., Fabiani, M., Mantulin, W. W., \& GratTON, E. (1994). Feasibility of intracranial near-infrared optical scanning. Psychophysiology, 31, 211-215.

Gray, C. M., Konig, P., ENGEL, A. K., \& Singer, W. (1989). Oscillatory responses in cat visual cortex exhibit inter-columnar synchronization which reflects global stimulus properties. Nature, 338, 334-337.

Grinvald, A., Lieke, E., Frostig, R. D., Gilbert, C. D., \& Wiesel, T. N. (1986). Functional architecture of cortex revealed by optical imaging of intrinsic signals. Nature, 324, 361-364.

HACKLEY, S. A. (1993). An evaluation of the automaticity of sensory processing using event-related potentials and brain-stem reflexes. Psychophysiology, 30, 415-428.

Hackley, S. A., \& Valle-Inclán, F. (1998). Autonomic alerting does not speed late motoric processes in a reaction time task. Nature, 391 , 786-788.

HAGLUND, M. M. (1997). Intraoperative optical imaging of epileptiform and functional activity. Neurosurgery Clinics of North America, 8, 413-420.

Haglund, M. M., Ojemann, G. A., \& Hochman, D. W. (1992). Optical imaging of epileptiform and functional activity in human cerebral cortex. Nature, 358, 668-671.

HARI, R., \& LOUNASMAA, O. V. (1989). Recording and interpretation of cerebral magnetic fields. Science, 244, 432-436.

Heinze, H. J., Mangun, G. R., Burchert, W., Hinrichs, H., Scholz, M., Munte, T. F., Gos, A., Scherg, M., Johannes, S., Hundeshagen, H., Gazzaniga, M. S., \& Hillyard, S. A. (1994). Combined spatial and temporal imaging of brain activity during visual selective attention in humans. Nature, 372, 543-546.

HILL, D. K., \& KEYNES, R. D. (1949). Opacity changes in stimulated nerve. Journal of Physiology, 108, 278-281.

Hirth, C., Obrig, H., Valdueza, J., Dirnagl, U., \& Villringer, A. (1997). Simultaneous assessment of cerebral oxygenation and hemodynamics during a motor task: A combined near infrared and transcranial Doppler sonography study. Advances in Experimental Medicine \& Biology, 411, 461-469.

Hock, C., Villringer, K., Muller-Spahn, F., Wenzel, R., Hefkeren, H., Schuh-Hofer, S., Hofmann, M., Minoshima, S., Schwaiger, M., Dirnagl, U., \& Villringer, A. (1997). Decrease in parietal cerebral hemoglobin oxygenation during performance of a verbal fluency task in patients with Alzheimer's disease monitored by means of near-infrared spectroscopy (NIRS) Correlation with simultaneous rCBF-PET measurements. Brain Research, 755, 293-303.

Hoshi, Y.. \& TAMURA, M. (1993). Dynamic multichannel near-infrared optical imaging of human brain activity. Journal of Applied Physiologv, 75, 1842-1846. 
Hubel, D. H., \& Wiesel, T. N. (1979). Brain mechanisms of vision Scientific American, 241(3), 150-162.

ISHIMARU, A. (1978). Wave propagation and scattering in random media (Vol. 1). New York: Academic Press.

Javitt, D. C., Schroeder, C. E., Steinschneider, M., Arezzo, J. C. \& VAUGHAN, H. G., JR. (1992). Demonstration of mismatch negativity in the monkey. Electroencephalography \& Clinical Neurophysiology, 83, 87-90.

Javitt, D. C., Steinschneider, M., Schroeder, C. E., Vaughan, H. G., \& AREzzo, J. C. (1994). Detection of stimulus deviance within primate primary auditory cortex: Intracortical mechanisms of mismatch negativity (MMN) generation. Brain Research, 667, 192-200.

Jiang, H., Paulsen, K. D., \& Osterberg, U. L. (1996). Optical image reconstruction using DC data: Simulations and experiments. Physics in Medicine \& Biology, 41, 1483-1498.

JoBSIS, F. F. (1977). Noninvasive, infrared monitoring of cerebral and myocardial oxygen sufficiency and circulatory parameters. Science, 198, 1264-1267.

Johnson, M. K., Nolde, S. F., Mather, M., Kounios, J., Schacter, D. L., \& CURRAN, T. (1997). The similarity of brain activity associated with true and false recognition memory depends on test format. Psychological Science, 8, 250-257.

Kato, T., Kamei, A., Takashima, S., \& Ozaki, T. (1993). Human visual cortical function during photic stimulation monitoring by means of near-infrared spectroscopy. Journal of Cerebral Blood Flow \& Metabolism, 13, 516-520.

Kelley, W. M., Buckner, R. L., Miezin, F. M., Raichle, M. E., Cohen, N. J., \& Petersen, S. E. (1998). Hemispheric specialization in human dorsal frontal cortex and medial temporal lobe for verbal and nonverbal memory encoding. Neuron, 20, 927-936.

KolB, B., \& Whishaw, I. Q. (1996). Fundamentals of human neuropsychology (4th ed.). New York: W. H. Freeman.

Kwong, K. K., Belliveau, J. W., Chesler, D. A., Goldberg, I. E. Weisskoff, R. M., Poncelet, B. P., Kennedy, D. N., Hoppel, B. E., Cohen, M. S., Turner, R., Cheng, H.-M., Brady, T. J., \& Rosen, B. R. (1992). Dynamic magnetic resonance imaging of human brain activity during primary sensory stimulation. Proceedings of the $\mathrm{Na}$ tional Academy of Sciences, 89, 5675-5679.

Lee, A. T., Glover, G. H., \& Meyer, C. H. (1995). Discrimination of large venous vessels in time-course spiral blood-oxygen-leveldependent magnetic-resonance functional neuroimaging. Magnetic Resonance in Medicine, 33, 745-754.

Loftus, E. F., \& Pickrell, J. E. (1995). The formation of false memories. Psychiatric Annals, 25, 720-725.

LORENTE DE Nó, R. (1947). Action potential of the motoneurons of the hypoglossus nucleus. Journal of Cellular \& Comparative Physiology, 29, 207-287.

LU, Z. L., Williamson, S. J., \& Kaufman, L. (1992). Behavioral lifetime of human auditory sensory memory predicted by physiological measures. Science, 258, 1668-1670.

MaloneK, D., \& GRINVAld, A. (1996). Interactions between electrical activity and cortical microcirculation revealed by imaging spectroscopy: Implications for functional brain mapping. Science, $\mathbf{2 7 2}$. 551-554.

Malonek, D., ToOtell, R. B., \& Grinvald, A. (1994). Optical imaging reveals the functional architecture of neurons processing shape and motion in owl monkey area MT. Proceedings of the Royal Society of London: Series B, 258, 109-119.

MARR, D. (1982). Vision. New York: W. H. Freeman.

MCCARTHY, G., \& WoOD, C. C. (1987). Intracranial recordings of endogenous ERPs in humans. Electroencephalography \& Clinical Neurophysiology, 39, 331-337.

MefK, J. H., Elwell, C. E., Khan, M. J., Romaya, J., Wyatt, J. S., DELPY, D. T., \& ZEKI, S. (1995). Regional changes in cerebral haemodynamics as a result of a visual stimulus measured by near infrared spectroscopy. Proceedings of the Roval Society of London: Series B, 261,351-356.

Menon, V., Freeman, W. J., Cutillo, B. A., Desmond, J. E., Ward, M. F., Bressler, S. L., Laxer, K. D., Barbaro, N., \& Gevins, A. S.
(1996). Spatio-temporal correlations in human gamma band electrocorticograms. Electroencephalography \& Clinical Neurophysiology, 98, 89-102. [Published erratum appears in Electroencephalography \& Clinical Neurophysiology, 1996, 98, 228.]

Mesulam, M. M. (1990). Large-scale neurocognitive networks and distributed processing for attention, language, and memory. Annals of Neurology, 28, 597-613.

Meyer, D. E., Osman, A. M., Irwin, D. E., \& Yantis, S. (1988). Modern mental chronometry. Biological Psychology, 26, 3-68.

Miltner, W., Johnson, R., JR., Simpson, G. V., \& Ruchin, D. S. (1994). A test of brain electrical source analysis (BESA): A simulation study. Electroencephalography \& Clinical Neurophysiology, 91, 295-310.

NÄÄTÄNEN, R. (1992). Attention and brain function. Hillsdale, NJ: Erlbaum.

NunEZ, P. L. (1981). Electric fields of the brain. New York: Oxford University Press.

Nyberg, L., Cabeza, R., \& Tulving, E. (1996). PET studies of encoding and retrieval: The HERA model. Psychonomic Bulletin \& Review, 3, 135-148

Obrig, H., Wolf, T., Doge, C., Hulsing, J. J., Dirnagl, U., \& VillRINGER, A. (1996). Cerebral oxygenation changes during motor and somatosensory stimulation in humans, as measured by near-infrared spectroscopy. Advances in Experimental Medicine \& Biology, 388, 219-224.

Okada, E., Firbank, M., \& Delpy, D. T. (1995). The effect of overlying tissue on the spatial sensitivity profile of near-infrared spectroscopy. Physics in Medicine \& Biology, 40, 2093-2108.

Okada, Y. C., Willlamson, S. J., \& Kaufman, L. (1982). Magnetic field of the human sensorimotor cortex. International Journal of Neuroscience, 17, 33-38.

PAulsen, K. D., \& JiAng, H. (1995). Spatially varying optical property reconstruction using a finite element diffusion equation approximation. Medical Physics, 22, 691-701.

Petersen, S. E., Corbetta, M., Miezin, F. M., \& Shulman, G. L. (1994). PET studies of parietal involvement in spatial attention: Comparison of different task types. Canadian Journal of Experimental Psychology, 48, 319-338.

PoSNer, M. I. (ED.) (1989). Foundations of cognitive science. Cambridge, MA: MIT Press.

Posner, M. I., \& Raichle, M. E. (1994). Images of mind. New York: Scientific American Books.

RaICHLE, M. E. (1986). Neuroimaging. Trends in Neurosciences, 9 , 525-529.

Raichle, M. E. (1994, April). Visualizing the mind. Scientific American, 270, 58-64.

Rector, D. M., Poe, G. R., Kristensen, M. P., \& Harper, R. M. (1995). Imaging the dorsal hippocampus: Light reflectance relationships to electroencephalographic patterns during sleep. Brain Research, 696, 151-160.

Rector, D. M., Poe, G. R., Kristensen, M. P., \& Harper, R. M. (1997) Light scattering changes follow evoked potentials from hippocampal Schaeffer collateral stimulation. Journal of Neurophysiology, $\mathbf{7 8}$, $1707-1713$

ReEs, G., Frith, C. D., \& Lavie, N. (1997). Modulating irrelevant motion perception by varying attentional load in an unrelated task. Science, 278, 1616-1619.

Reppas, J. B., Niyogi, S., Dale, A. M., Sereno, M. I., \& Tootell, R. B. (1997). Representation of motion boundaries in retinotopic human visual cortical areas. Nature, 388, 175-179.

Riehle, A., Gruen, S., Diesmann, M., \& Aertsen, A. (1997). Spike synchronization and rate modulation differentially involved in motor cortical function. Science, 278, 1950-1953.

RIEHLE, A., \& REQUin, J. (1993). The predictive value for performance speed of preparatory changes in neuronal activity of the monkey motor and premotor cortex. Behavioural Brain Research, 53, 35-49.

Ritter, W., Deacon, D., Gomes, H., Javitt, D. C., \& Vaughan, H. G., JR. (1995). The mismatch negativity of event-related potentials as a probe of transient auditory memory: A review. Ear \& Hearing, 16. 52-67. 
RoEdigER, H. L., III (1996). Memory illusions. Journal of Memory \& Language, 35, 76-100.

Roediger, H. L., III, \& MCDermott, K. B. (1995). Creating false memories: Remembering words not presented in lists. Journal of Experimental Psychology: Learning, Memory, \& Cognition, 21, 803-814

Roelfsema, P. R., Engel, A. K., Konig, P., \& Singer, W. (1996). The role of neuronal synchronization in response selection: A biologically plausible theory of structured representations in the visual cortex Journal of Cognitive Neuroscience, 8, 603-625.

Roy, C. S., \& Sherrington, C. S. (1890). On the regulation of the blood supply to the brain. Journal of Physiology, 11, 85-108.

RuGG, M. D. (ED.) (1997). Cognitive neuroscience. Cambridge, MA MIT Press.

RuGG, M. D., \& Coles, M. G. H. (EDs.) (1995). Electrophysiology of mind: Event-related brain potentials and cognition. Oxford: Oxford University Press.

SANDERS, A. F. (1981). Stage analysis of reaction processes. In G. E. Stelmack \& J. Requin (Eds.), Tutorials in motor behavior (pp. 331-354) Amsterdam: North-Holland.

Schacter, D. L., Reiman, E., Curran, T., Yun, L. S., Bandy, D., MCDermott, K. B., \& RoEdiger, H. L., III (1996). Neuroanatomical correlates of veridical and illusory recognition memory: Evidence from positron emission tomography. Neuron, 17, 267-274

Scherg, M., Vajsar, J., \& Picton, T. W. (1989). A source analysis of the human auditory evoked potentials. Journal of Cognitive Neuroscience, 1, 336-355.

Scherg, M., \& Von Cramon, D. (1986). Evoked dipole source potentials of the human auditory cortex. Electroencephalography \& Clinical Neurophysiology, 65, 344-360

Sereno, M. I., Dale, A. M., Reppas, J. B., Kwong, K. K., Belliveau, J. W., Brady, T. J., Rosen, B. R., \& ToOtell, R. B. (1995). Borders of multiple visual areas in humans revealed by functional magnetic resonance imaging. Science, $\mathbf{2 6 8 , 8 8 9 - 8 9 3}$

SINGER, W. (1994). Coherence as an organizing principle of cortical functions. In O. Sporns \& G. Tononi (Eds.), Selectionism and the brain (Vol. 37, pp. 153-183). San Diego, CA: Academic Press.

Skarda, C. A., \& Freeman, W. J. (1987). How brains make chaos in order to make sense of the world. Behavioral \& Brain Sciences, 10, 161-195.

SQUiRE. L. R. (1989). Mechanisms of memory. In K. L. Kelner \& D. E. Koshland (Eds.), Molecules to models: Advances in neuroscience (chap. 40). Washington, DC: AAAS

Stepnoski, R. A., La Porta, A., Raccuia-Behling, F, Blonder, G. E. , Slusher, R. E., \& Kleinfeld, D. (1991). Noninvasive detection of changes in membrane potential in cultured neurons by light scattering. Proceedings of the National Academy of Sciences, $\mathbf{8 8}$ 9382-9386

SternBerg, S. (1966). High-speed scanning in human memory. Science, 153, 652-654

STERNBERG, S. ( 1969$)$. The discovery of processing stages: Extensions of Donders' method. In W. G. Koster (Ed.), Attention and performance II (pp. 276-315). Amsterdam: North-Holland.

Stevenson, D. K., Vreman, H. J., \& Benaron, D. A. (1996). Evaluation of neonatal jaundice: Monitoring the transition in bilirubin metabolism. Journal of Perinatology, 16(3. Pt. 2), S62-67.

Taddeucci, A., Martelli, F., Barilli, M., Ferrari, M., \& ZacCANTI, G. (1996). Optical properties of brain tissue. Journal of Biomedical Optics, 1, 117-123.

Tamura, M., Hoshi, Y., \& OKada, F. (1997). Localized near-infrared spectroscopy and functional optical imaging of brain activity. Philosophical Transactions of the Roval Society of London: Series B, 352 , 737-742.

Thatcher, R. W., Toro, C., Pflieger, M. E., \& Hallett, M. (1994). Human neural network dynamics using multimodal registration of EEG, PET, and MRI. In M. Hallett, T. A. Zeffiro, E. R. John, \& M. Huerta (Eds.), Functional neuroimaging: Technical foundations (pp. 269-278). San Diego, CA: Academic Press

Tittinen, H., Alho, K., Huotilainen, M., Ilmoniemi, R. J., SimOL.A, J., \& NÄÄTÄNEN, R. (1993). Tonotopic auditory cortex and the magnetoencephalographic (MEG) equivalent of the mismatch negativity. Psichophisiology, 30, 537-540.
Toga, A. W., \& Mazziotta, J. C. (Eds.) (1996). Brain mapping: The methods. San Diego, CA: Academic Press.

Tootell, R. B., Dale, A. M., Sereno, M. I., \& Malach, R. (1996). New images from human visual cortex. Trends in Neurosciences, 19 , 481-489.

Tulving, E., Kapur, S., Craik, F. I., Moscovitch, M., \& Houle, S. (1994). Hemispheric encoding/retrieval asymmetry in episodic memory: Positron emission tomography findings. Proceedings of the $\mathrm{Na}$ tional Academy of Sciences, 91, 2016-2020.

TURNER, R. (1995). Functional mapping of the human brain with magnetic resonance imaging. Seminars in the Neurosciences, 7, 179-194.

VILLRINGER, A. (1997). Understanding functional neuroimaging methods based on neurovascular coupling. Advances in Experimental Medicine \& Biology, 413, 177-193.

Villringer, A., \& Chance, B. (1997). Non-invasive optical spectroscopy and imaging of human brain function. Trends in Neurosciences, 20, 435-442.

VILlRINGER, A., \& DirnaGl, U. (1995). Coupling of brain activity and cerebral blood flow: Basis of functional neuroimaging. Cerebrovascular \& Brain Metabolism Reviews, 7, 240-276.

Wang, G., Tanaka, K., \& Tanifuji, M. (1996). Optical imaging of functional organization in the monkey inferotemporal cortex. Science, 272, 1665-1668.

Weinberg, H., Cheyne, D., \& Crisp, D. (1990). Electroencephalographic and magnetoencephalographic studies of motor function. $A d$ vances in Neurology, 54, 193-205.

Wenzel, R., Obrig, H., Ruben, J., Villaringer, K., Thiel, A., Bernarding, J., DiRnagl, U., \& VILLRINGER, A. (1996). Cerebral blood oxygenation changes induced by visual stimulation in humans. Journal of Biomedical Optics, 1, 399-404.

Wilson, B. C., Patterson, M. S., Flock, S. T., \& Wyman, D. R. (1989). Tissue optical properties in relation to light propagation models and in vivo dosimetry. In B. Chance (Ed.), Photon migration in tissues (pp. 25-42). New York: Plenum.

Winkler, I., Tervaniemi, M., Huotilainen, M., Ilmoniemi, R., Ahonen, A., Salonen, O., Standertskjold-Nordenstam, C. G., \& NÄÄTÄNEN, R. (1995). From objective to subjective: Pitch representation in the human auditory cortex. NeuroReport, 6, 2317-2320.

Zhu, W., Wang, Y., Deng, Y., Yao, Y., \& Barbour, R. L. (1997). A wavelet-based multiresolution regularized least squares reconstruction approach for optical tomography. IEEE Transactions on Medical Imaging, 16, 210-217.

Zhu, W., Wang, Y., Yao, Y., Chang, J., Graber, H. L., \& Barbour, R. L. (1997). Iterative total least-squares image reconstruction algorithm for optical tomography by the conjugate gradient method. Journal of the Optical Society of America A, 14, 799-807.

\section{NOTES}

1. PET is a functional imaging method designed to measure noninvasively the concentration of radioactively labeled substances into parts of the body and, in particular, parts of the brain. Briefly, the methodology involves (1) introducing the radioactive substance into the body, (2) waiting an interval to allow the substance to diffuse to the target organ, and (3) imaging the distribution of the substance in the organ. Depending on the type of isotope used, the nature of the substance in which the isotope is incorporated, and the procedures used for administering the substance, PET will have different functional, temporal, and spatial properties. The most commonly used procedures used to obtain PET data from the human brain are deoxyglucose and $0^{15}$-PET. The first is a measure of brain metabolism, and it requires a long interval between injection and data acquisition. During this interval, cognitive tasks are usually performed. The second method is much faster, and it provides a measure of blood flow in active brain areas.

2. MRI is a noninvasive procedure to image some of the magnetic properties of different parts of the body, including the brain. The BOLD-fMRI signal is based on localized changes in magnetic properties that depend on variations in the relative concentration of oxyhemoglobin and deoxyhemoglobin. These changes are thought to occur because of changes in blood flow (and corresponding changes in the influx 
of oxygenated blood) brought forth by brain activity. fMRI images can be obtained quickly (typically every few seconds).

3. The average ERP is often analyzed into its "components," which can be broadly defined as segments of the waveforms that covary in response to experimental manipulations and show a consistent latency and scalp distribution across experiments and conditions (Fabiani et al., in press). The concept of component, although very useful in the analysis of ERP effects, has been largely debated because (1) components often overlap in time (and space), and (2) it is often difficult to attribute a given effect exclusively to a given component.

4. However, an advantage of ERP recording (with respect to MEG) is its lower cost (less than $\$ 100,000$ for a full-head ERP recording system vs. $\$ 2$ million for a full-head MEG system).

5. Malonek and Grinvald's (1996) study and previous papers on optical imaging of the exposed cortex (see Bonhoeffer \& Grinvald, 1996 for a review) also identify another type of functional response located in the large blood vessels--in particular, veins draining blood from active brain areas. This response may be less localized, because the relationship between large blood vessels and functionally active brain areas is not very tight. Thus, increases in blood flow may be observed at a substantial distance from the active regions of the brain. The relative impact of small and large blood vessels on the effects visualized by hemodynamic imaging methods (the "brain or vein" issue) is variable and depends on how the signal is measured. Villringer and Dirnagi (1995) argue that the BOLD-fMRI signal appears to be heavily weighted toward very small blood vessels (although this does in fact depend on voxel size, pulse sequence, and analysis strategy; see Lee, Glover, \&
Meyer, 1995). Therefore, under optimal recording conditions, BOLDfMRI possesses a good localization power (within the limits described for the slow oxygenation signal described by Malonek \& Grinvald, 1996). Note, however, that other fMRI signals may have different localization values. For PET measures, the localization power is partially dependent on the contrast agent used and the procedure adopted (Villringer \& Dirnagl, 1995, and note 1).

6 . The amount of energy used for EROS recordings is very small (less than $1 \mathrm{~mW}$ ) and not perceivable by the subject.

7. Note, however, that the distinction between different types of signal (if needed) could profit from the use of multiple wavelengths.

8 . This result should not be taken to indicate that, in general, the response in extrastriate area should have a latency of 200-300 msec. Indeed, as shown in subsequent studies, responses in extrastriate areas were observed at latencies of $100 \mathrm{msec}$ or less, albeit under different conditions.

9. The expected location of area V1 was determined as the point of maximum EROS response independent of condition. fMRI data available for one subject provided a convergent validation for this approach. However, only histological data can provide conclusive determination of the location of $\mathrm{VI}$.

10. These memory effects are not inconsistent with the results of the attention experiment, showing that activity in area V1 is unaffected by attention manipulation. In fact, complex memory effects can occur outside the control of attention (e.g., implicit and sensory memory) and are also evident for other brain phenomena (e.g., mismatch negativity component of the ERP). 


\section{APPENDIX}

\section{Main Absorbers in the Human Brain}

Whereas exposed cortex measures can be conducted using light of any wavelengths, only NIR light (wavelength $=700-1,000 \mathrm{~nm}$ ) penetrates head tissues enough to make noninvasive measures (i.e., measures recorded with the skull and skin intact) practical. The absorption spectra of a few major absorbers (i.e., substances that absorb light) are shown in Figure Al (Wilson, Patterson, Flock, \& Wyman, 1989).

From this figure, it is evident that water (which is a large component of most living tissues) absorbs a substantial amount of light with wavelengths longer than $1,000 \mathrm{~nm}$ (as well as in the ultraviolet range). In addition, hemoglobin absorbs light in the visible range, but its absorption drops considerably in the NIR region (although it is still measurable). It should also be noted that oxyhemoglobin and deoxyhemoglobin have somewhat different absorption spectra, both in the visible region and in the NIR region. This difference in absorption spectra makes it possible to measure separately the concentrations of oxyhemoglobin and deoxyhemoglobin in living tissues using spectroscopic methods (both in the visible range and in the NIR range). The other main absorber shown in Figure Al is melanin. The absorption spectrum of melanin and its pattern of distribution vary among individuals, as a function of genetic (e.g., skin and hair color, etc.) and other factors. This protein is particularly concentrated in hair. Therefore, noninvasive measurements are best taken with hair

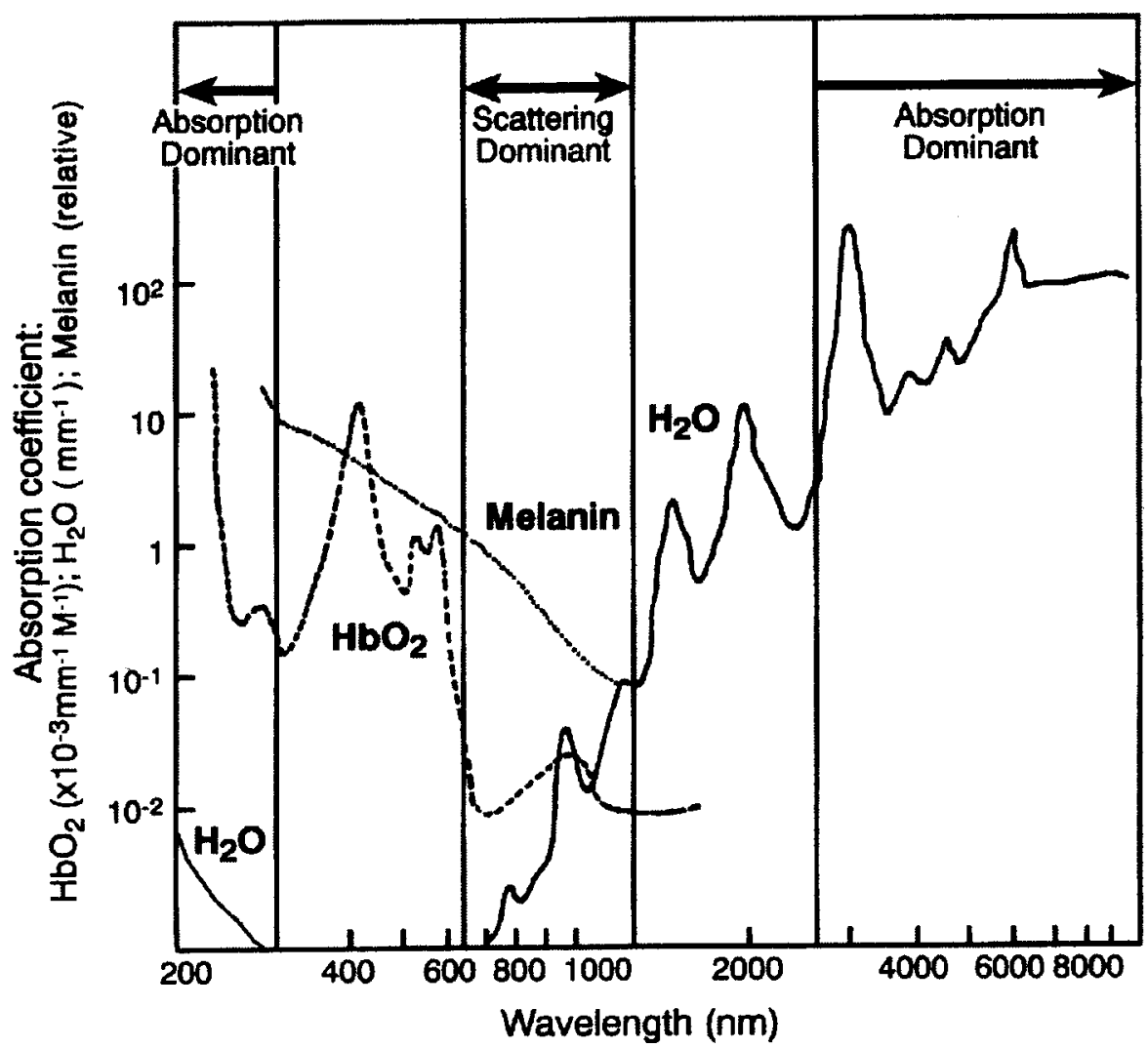

Figure A1. Absorption spectra for three significant physiological absorbants (water, oxyhemoglobin, and melanin) in a wavelength range between the ultraviolet (left) and the infrared (right). The absorption spectrum of another significant absorbant (deoxyhemoglobin) is not shown, but it is similar that of oxyhemoglobin, with enough discrepancies to allow the distinction of these two substances. Note that water absorbs heavily in the ultraviolet $(<300 \mathrm{~nm})$ and far-infrared $(>1,000 \mathrm{~nm})$ ranges. This limits the wavelength range viable for use in noninvasive optical studies of the human brain. This range is further limited by the high absorption of hemoglobin in the visible range $(400-700 \mathrm{~nm})$. Therefore, the best penetration into the human body is obtained within the near-infrared range $(700-1,000 \mathrm{~nm})$. Within this range, the main obstacle to light penetration is due to scattering. Note-From "Tissue Optical Properties in Relation to Light Propagation Models and In Vivo Dosimetry," by B. C. Wilson, M. S. Patterson, S. T. Flock, and D. R. Wyman, 1989, in B. Chance (Ed.), Photon Migration in Tissues (p. 28). Copyright 1989 by Plenum Press. Reprinted with permission. 
combed away and are more easily obtained in subjects with light and/or thin hair. However, the color of the skin does not appear to make a substantial difference (E. Gratton, personal communication, May 15, 1995).

\section{The Physical Bases of NIRS}

The concentration of a substance in a solution can be derived using the modified Lambert-Beer law:

$$
A=\varepsilon \times c \times d \times B+G,
$$

where $A$ is the attenuation of light, $\varepsilon$ is the absorption coefficient of the substance, $c$ is the substance concentration, $d$ is the size of the sample being studied (or the distance between source and detector), $B$ is a coefficient related to the prolongation of the photon path due to scattering, and $G$ is the signal loss due to scattering independent of absorption. All of these factors except $c$ and $d$ are also wavelength dependent.

Note that the concentration of a pure substance in a nonscattering medium can be derived using a single wavelength. However, when multiple substances are present in the medium, more than one wavelength is required to derive the concentration of each of them (as a minimum, as many wavelengths are required as there are absorbing substances in the medium, provided that they differ in their absorption spectra). While this may generate a difficult problem for in vivo applications (where the number of different substances may be very high), it is usually possible to consider only a small subset of substances whose concentration and/or absorption properties are particularly large. The two main absorbers of NIR light in the human body are oxyhemoglobin and deoxyhemoglobin. Therefore, it is possible to measure the absolute concentration of these two substances with a fair degree of accuracy with only a few wavelengths. In fact, several companies are marketing devices to measure noninvasively the concentration of these substances in the human body based on the Lambert-Beer law. These devices are called optical tissue oxymeters, and they may cost from a few hundred dollars up to several thousands. These measures have some level of spatial specificity, in that the area explored by the measures is limited to that surrounding the recording instrument (a few centimeters in each direction), because of the limited penetration of light in head tissue. As we will see later, their spatial specificity can be greatly improved with appropriate methodologies.

It should be noted, however, that these measures should be considered only as estimates. This is so for several reasons. First, they depend on the assumption that only very few and well-known absorbers (such as oxyhemoglobin and deoxyhemoglobin) should be considered (and all the others can be safely ignored). Second, they depend on the assumption that the average length of the trajectory followed by photons within the body (length of the photon path) is known. This is because the light attenuation associated with the concentration of a particular substance depends on the distance traveled by the light within the solution under study (e.g., the thicker the medium, the more light attenuation will occur even if the concentration remains constant). However, in a scattering medium (such as the head or other parts of the human body), the path followed by photons is difficult to compute, and it is largely dependent on the scattering properties of the medium itself. In NIRS studies, the photon-path length is usually estimated on the basis of previous studies performed on similar structures.

The third assumption is that the medium or solution used for NIRS should be homogeneous. However, the head (and other parts of the human body) is largely dishomogenous, with different areas varying in terms of their scattering and absorption coefficients. This complicates the measurement of the photon path and, therefore, of the average photon-path length. As a consequence, all the observations obtained with this procedure are only "average" measures over an extended volume. Several methodologies are now being developed to aid in the computation of the path followed by photons, as well as to yield a 3-D reconstruction of the scattering and absorption properties of dishomogenous media.

\section{Time-Domain and Frequency-Domain Optical Methods}

Measurements of the length of the photon path require techniques that quantify the time taken by photons to travel through tissue. This quantification requires a source of light that is modulated in intensity (timeresolved measures). This may be accomplished by having very short pulses of light (time-domain methods) or by modulating the light at a very high frequency (e.g., $>100 \mathrm{MHz}$; frequency-domain methods).

A disadvantage of the time-domain approach is that acquisition of a sufficient number of photons for the derivation of useful measures is relatively slow. In addition, this technology may be expensive because of the electronics and optics required. In contrast, frequency-domain methods are less expensive and allow for a faster data acquisition. E. Gratton, Jameson, Rosato, and Weber (1984; see also E. Gratton \& Limkeman, 1983 ) developed a procedure, based on the use of a cross-correlation frequency, which allows the use of a lowfrequency data acquisition system, based on a personal computer and a dedicated analog/digital card (which are relatively inexpensive) to acquire time-resolved optical data.

An additional advantage of time-resolved optical methods is that they can be used to improve the spatial specificity (and the spatial resolution) of the measures. This is achieved by selecting a subset of the photons emitted from the source that reach the detector and that have a relatively short time of flight (i.e., time required to travel between the source and the detector). Alfano, Demos, and Gayen (1997) showed that photons with a short time of flight follow relatively similar trajectories within a medium. For this reason, these photons tra- 


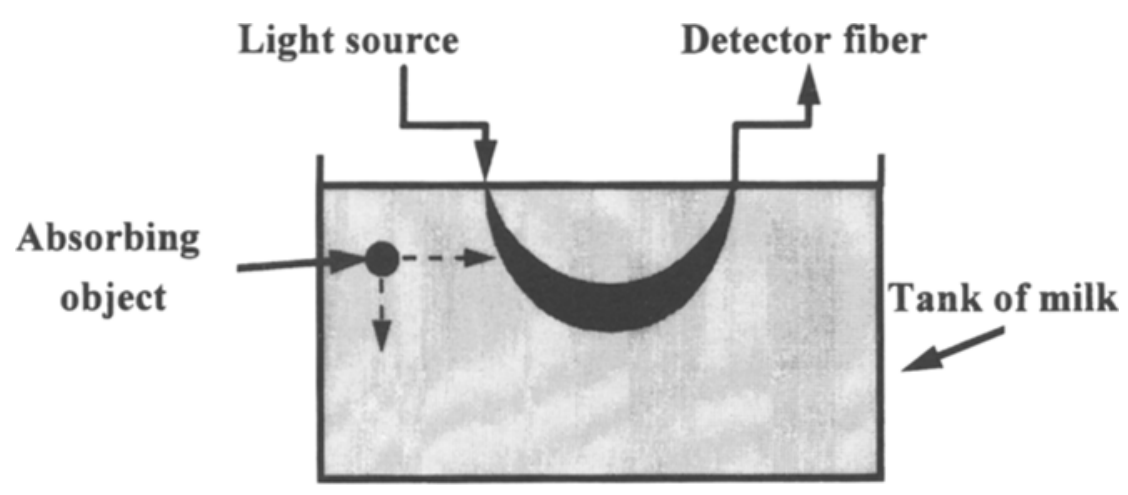

Figure A2. Schematic representation of the apparatus used for the phantom measurements of the path of light. Measurements of photon migration parameters were taken using a light source and a detector located at the surface of a tank full of skim milk. This phantom was used to simulate a source and a detector located on the surface of the head, which, like skim milk, scatters NIR light. A very small absorbing sphere (represented here on a much larger scale) was immersed in the milk. The sphere was moved to scan a large number of different positions inside the tank. When the object intercepted (and absorbed) photons that moved between the source and the detectors, it interfered with the measurement of the photon migration parameters. Therefore, the volume explored (path of light) can be determined on the basis of the locations at which the absorbing sphere interfered with the measurements.

verse a relatively small volume of tissue, and measurements taken using photons selected in this fashion possess good spatial specificity.

Note that, by selecting photons with increasingly short times of flight, it is possible to obtain photon paths that are increasingly coherent. This way we can obtain measures with very high spatial specificity (i.e., on the order of microns). However, this requires the selection of a smaller and smaller proportion of the photons, which would result in a decreased signal-to-noise ratio. At some point, the number of photons reaching a particular detector may be so small as to be unmeasurable, and, thus, there exists a practical limit to the spatial resolution that can be obtained in this fashion. In fact, resolutions higher than $1 \mathrm{~mm}$ could be difficult to achieve in noninvasive studies.

\section{Path of Light}

The phantom study reported by G. Gratton et al. (1994) was conducted by measuring the transmission of NIR light within a tank full of a scattering substance (skim milk). Both the light source and the detector were placed on the surface of the milk, $7 \mathrm{~cm}$ apart from each other (see Figure A2). This arrangement simulated situations obtained for head measurements, in which sources and detectors are also located on the surface of an air-bounded, scattering medium. The path followed by the light was measured by placing a small ( $3 \mathrm{~mm}$ in diameter) absorbing sphere inside the milk at a large number of different locations. The extent to which the sphere placed at any particular location influenced the measurement was used to determine whether the location was part of the path followed by the light. The results of this study (presented in Figure A3) confirmed the prediction that the path followed by NIR photons is indeed crescent-shaped, reaching a maximum depth approximately equal to half the distance between the source and the detector.

Figure A 3 shows that, for measures of photon delay, the thickness of the path is only a fraction $(<1 / 5)$ of the distance between the source and the detector. This occurs because the measures were obtained using a frequency-domain approach that allowed for the selection of photons with relatively uniform times of flight (see section 3, above).

\section{Optical Properties of Head Layers}

The medium used in the phantom experiment described above was relatively homogenous, whereas the head can be expected to be much less so. In particular, several layers can be identified with very different optical properties:

(1) Hair. Because of the presence of melanin, hair may have a very high absorption coefficient (depending on its color). Fortunately, it is possible to use sources and detectors that are quite small, so as to allow measurements to be taken between the hair.

(2) Skin. This layer is quite thin and its absorption and scattering coefficients are moderate, thus allowing a good penetration of NIR light. 

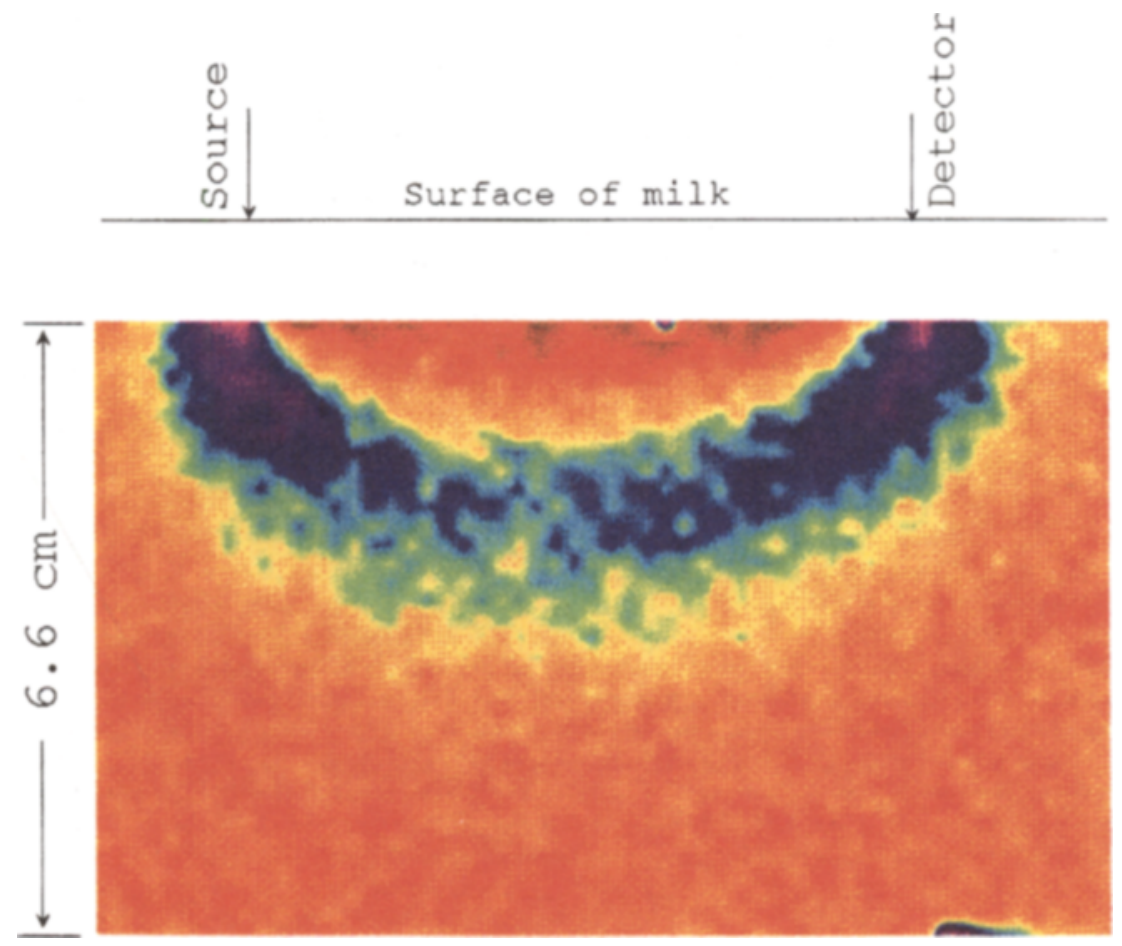

Figure A3. Results of the phantom experiment on the measurement of the path of light. The graph shows a map of the effect of the absorbing sphere on the photon phase-delay parameter, as a function of its position. Locations mapped in red correspond to sphere positions where no effect was observed with respect to conditions in which there was no sphere in the medium. These are locations outside the volume explored by the procedure. Locations mapped in blue correspond to sphere positions in which an effect was observed with respect to conditions in which there was no sphere in the medium. These are locations within the volume explored by the procedure. Note-From "Feasibility of Intracranial Near-Infrared Optical Scanning," by G. Gratton, J. S. Maier, M. Fabiani, W. W. Mantulin, and E. Gratton, 1994, Psychophysiology, 31, p. 213. Copyright 1994 by Cambridge University Press. Reprinted with permission.

(3) Skull. This layer has a relatively high scattering coefficient but a very low absorption coefficient. Therefore, photons can penetrate this layer relatively easily. This was demonstrated in another experiment reported by G. Gratton et al. (1994), in which an absorbing object was clearly detected even if placed within a sheep skull (both the absorbing object and the skull were submerged into skim milk).

(4) Meninges and cerebrospinal fluid. These layers are thin and transparent to light, posing no significant obstacle to the movement of photons.

(5) Cortex. The cortex has moderate scattering and absorption coefficients and can therefore be penetrated by NIR light (Taddeucci, Martelli, Barilli, Ferrari, \& Zaccanti, 1996).

(6) White matter. The white matter has a very high scattering coefficient (Taddeucci et al., 1996), and it is therefore unclear whether NIR photons can penetrate deeply into this tissue. Most of the photons might be reflected back to the previous layer, which is consistent with the fact that this tissue is white and lucid when seen with the naked eye.

As a consequence of the dishomogeneity of the different layers of the head, the path of the photons may be distorted with respect to the picture presented in Figure A3: Photons will tend to travel along the cortical layer, which is "sandwiched" between the skull and the white matter (both layers with high scattering coefficients). For this reason, head measures of photon delay are essentially cortical measures.

\section{3-D Reconstruction of Optical Data}

Most studies reported in this paper focus on the effects that scattering and absorption changes can have on surface measures. To increase the spatial specificity of the measure, it may be useful to derive 3-D reconstructions of the absorption and scattering coefficients of specific cortical areas. Several investigators have proposed methods for 3-D reconstruction (Arridge \& Hebden, 1997; Arridge \& Schweiger, 1997; Benaron, 
Ho, Spilman, Van Houten, \& Stevenson, 1994; Chang, Graber, \& Barbour, 1997; Chang, Graber, Koo, et al., 1997; Jiang, Paulsen, \& Osterberg, 1996; Paulsen \& Jiang, 1995; Tamura, Hoshi, \& F. Okada, 1997; Zhu, Wang, Deng, Yao, \& Barbour, 1997; Zhu, Wang, Yao, et al., 1997). The problem is easily tractable in relatively homogenous media (for which good 3-D reconstruction algorithms are available), but it is more complicated in dishomogenous media (such as the head). There are two main obstacles: (1) In dishomogenous media, the path followed by the photons depends on the scattering and absorption properties of the tissue, which are not known a priori and must be determined on the basis of the measures themselves. This makes the problem somewhat circular, because knowledge of the path is essential to determine the scattering and absorption coefficients. There are two approaches (not mutually exclusive) to this issue. The first is based on assigning approximate absorption and scattering coefficients (derived from the existing literature) to different areas identified on the basis of structural MRI images. The second is based on an iterative process, which provides temporary solutions that are then refined on subsequent iterations. (2) It is sometimes difficult to distinguish between scattering and absorption effects. This is in part due to the fact that an increase in scattering in a certain area may also lead to an increase in absorption in the very same areas, because a photon that "bounces" more times has a greater probability of being absorbed. In this respect, scattering and absorption are not independent phenomena.

Notwithstanding these difficulties, 3-D reconstruction algorithms (which are expected to provide a localization power of $1 \mathrm{~mm}$ or less) have been proposed for dishomogenous media. Some of these methods are based on more complex schemes involving the simultaneous use of multiple sources and/or detectors for each voxel (Chance, Kang, He, Weng, \& Sevick, 1993).

Most of the 3-D algorithms mentioned above require measurement from a large number of sources and detectors (i.e., more than 100). However, most of the EROS experiments reviewed in the present paper are based on a recording system with relatively few sources and detectors (less than 16). Thus, no 3-D reconstruction was attempted, and the spatial resolution is limited to the spatial sampling of the surface measures (approximately $5 \mathrm{~mm}$ ). 